\title{
High-fidelity non-linear analysis of metal sandwich panels
}

\author{
Alexander N. Nordas MEng, MSc, DIC \\ PhD candidate, Department of Civil and Environmental Engineering, \\ Imperial College, London, UK (corresponding author: \\ alexandros.nordas13@imperial.ac.uk) \\ Luis Santos MEng \\ PhD candidate, Department of Civil and Environmental Engineering, \\ Imperial College, London, UK
}

\author{
Bassam A. Izzuddin BEng, MSc, DIC, PhD, FIStructE \\ Professor of Computational Structural Mechanics, Department of Civil and \\ Environmental Engineering, Imperial College, London, UK \\ Lorenzo Macorini PhD \\ Senior Lecturer in Structural Engineering, Department of Civil and \\ Environmental Engineering, Imperial College, London, UK
}

The considerably superior specific strength and stiffness of sandwich panels in relation to conventional structural components makes their employment for two-way spanning structural applications a highly attractive option. An effective high-fidelity numerical modelling strategy for large-scale metal sandwich panels is presented in this paper, which enables the capturing of the various forms of local buckling and its progression over the panel domain, alongside the effects of material non-linearity and the spread of plasticity. The modelling strategy is further enhanced with a novel domain-partitioning methodology, allowing for scalable parallel processing on high-performance computing distributed memory systems. Partitioned modelling achieves a substantial reduction of the wall-clock time and computing memory demand for extensive non-linear static and dynamic analyses, while further overcoming potential memory bottlenecks encountered when conventional modelling and solution procedures are employed. A comparative evaluation of the speed-up achieved using partitioned modelling, in relation to monolithic models, is conducted for different levels of partitioning. Finally, practical guidance is proposed for establishing the optimal number of partitions offering maximum speed-up, beyond which further partitioning leads to excesses both in the non-linear solution procedure and the communication overhead between parallel processors, with a consequent increase in computing time.

\section{Notation}

$\boldsymbol{d}$ translational field vector of shell finite elements (FEs) in the global coordinate system

$\mathrm{NR}_{p} \quad$ nodal ratio for a partitioned model with $(p)$ child partitions

$\boldsymbol{r} \quad$ rotational field vector of shell FEs in the local coordinate system

$\overline{\boldsymbol{r}} \quad$ rotational field vector of shell FEs in the global coordinate system

$\mathrm{SF}_{p} \quad$ speed-up factor achieved by a partitioned model with $(p)$ child partitions

$\boldsymbol{t}$ translational field vector of shell FEs in the local coordinate system

$\boldsymbol{\alpha}_{\mathrm{h}} \quad$ hierarchic corrective parameters vector

$\boldsymbol{\alpha}_{\mathrm{o}} \quad$ objective parameters vector

$\varepsilon^{\mathrm{b}} \quad$ conforming bending strain vector of shell FEs in the local coordinate system

$\varepsilon^{\mathrm{m}} \quad$ conforming membrane strain vector of shell FEs in the local coordinate system

$\varepsilon^{s} \quad$ conforming shear strain vector of shell FEs in the local coordinate system

$\boldsymbol{\Psi}_{\mathrm{h}} \quad$ hierarchic corrective strain mode vector

$\boldsymbol{\Psi}_{\mathrm{o}} \quad$ objective strain mode vector

\section{Introduction}

Sandwich panels can be classified as composite structural components, comprising two thin and stiff plates connected to a structural core of a discrete geometric configuration by way of adhesion or welding. The core provides essential versatility to the composite mechanical response by maintaining a constant distance between the plates, thereby enhancing the flexural stiffness, while further serving as the principal load-transferring and shear-resistance mechanism. Due to the discrete configuration of the core, its beneficial action comes at a minimal weight trade-off, allowing sandwich panels to attain considerably enhanced stiffness and strength characteristics in comparison to solid components of equivalent weight. The mechanical response characteristics of sandwich composites are primarily dictated by the choice of material and the core topology. In the vast majority of weight-critical applications, composite materials of intrinsically low density, such as fibrereinforced polymers (FRP), fibre-reinforced glass (FRG) and metal composites, are usually employed in order to enhance further the already substantial weight-saving potential (Plantema, 1966). However, in large-scale applications, such as bridge construction, deck systems and ship vessels, the magnitude and type of applied loading dictates the use of 
higher-density materials with superior strength, mainly reinforced or prestressed concrete and structural steel. A long list of core topologies can be found in the literature, which is considerably condensed when the focus is shifted to large-scale structural applications comprising all-metal sandwich panels. Cellular or honeycomb and corrugated prismatic are among the prevailing core configurations for this application class, with the former achieving a superior two-way spanning response due to the core symmetry and homogeneity.

In sandwich panels with honeycomb core configurations, the cell geometry and orientation perpendicular to the panel plates result in the formation of discrete laterally unrestrained plated components of substantial slenderness, both at the cell walls and at the plate domains. These components are prone to elastoplastic compressive and shear buckling, which, alongside potential stress concentrations arising from the discrete nature of the core, interact with global flexural and/or shear failure modes, leading to highly diverse and complex stress and deformation states throughout the panel domain. The establishment of reliable estimates for the critical buckling load of individual plated components, as well as the resulting reduction in the panel global capacity, is therefore rendered highly cumbersome for large-scale systems subject to complex loading conditions. The sensitivity of the aforementioned estimates to geometric constraints imposed by the cell wall interactions and the welded connections, as well as to the magnitude and sense of imperfections and defects, further complicate the assessment of local buckling and the overall response characteristics of the sandwich panel. Taking the above into consideration, it is evident that an accurate global non-linear response evaluation calls for an increased level of modelling sophistication.

Several researchers have investigated the mechanical response of honeycomb-core sandwich panels numerically, analytically and experimentally, with the fundamental theoretical principles outlined in the books by Plantema (1966), Allen (1969) and Zenkert (1995). Zhang and Ashby (1992) and Gibson and Ashby (1999) established analytical formulations for the elastic properties and moduli of honeycomb-core sandwich composites and validated these against experimental and numerical results. Analytical estimates of the ultimate flexural, shear and buckling capacity of metal rectangular- and hexagonalhoneycomb-core structures and small-scale panels have also been established and validated against experimental results and finite-element (FE) models (Kee Paik et al., 1999; Zok et al., 2005). Liang and Chen (2006), alongside López Jiménez and Triantafyllidis (2013), established theoretical methodologies for the critical buckling load evaluation for honeycomb core configurations, while Cote et al. (2004) experimentally investigated the buckling response of metal honeycomb core topologies. Shifting the focus to large-scale metal laser-welded sandwich panels, publications are mainly limited to I-core configurations, where the failure modes and instabilities in ultimate conditions having been extensively investigated by Kolsters and Zenkert (2006a, 2006b, 2010), while a complete experimental and numerical study on the aforementioned configurations can be found in the SAND Core practice guide for marine applications (CORe, SAND, 2013).

Notwithstanding the significant research outlined above, the detailed non-linear analysis of large-scale metal sandwich panels with honeycomb core topologies has received much less attention, largely due to the associated modelling complexity and high computational demand posed by such intricate structures. With this backdrop, a high-fidelity non-linear FE modelling strategy is proposed in this paper, the key features of which are: (i) a highly accurate geometric modelling process for the core and plates, accounting for manufacturing and connection characteristics between individual cells, alongside initial imperfections; (ii) an extensive discretisation of the panel domain with geometrically non-linear shell finite elements (FEs), allowing for the effects of local buckling on the global panel response to be accurately captured; (iii) the employment of detailed non-linear material constitutive models capable of accurately capturing local yielding and the spread of plasticity over the domain, including hardening and strain-rate effects; and (iv) utilisation of a powerful domain partitioning on HPC that achieves considerable computational efficiency and overcomes memory bottlenecks.

The paper proceeds with presenting the main components of the proposed modelling strategy, including the elements employed for high-fidelity modelling and the application of the partitioning approach to sandwich panels with honeycomb cores. Two application studies are finally presented to illustrate the complex response characteristics of such sandwich panels as obtained from high-fidelity modelling, and to demonstrate the considerable savings in wall-clock time that can be achieved with the adopted partitioned modelling approach.

\section{High-fidelity modelling of all-metal sandwich panels}

For the domain discretisation of sandwich panels with rectangular and hexagonal honeycomb core topologies, ninenoded quadrilateral (Izzuddin and Liang, 2017) and six-noded triangular (Izzuddin and Liang, 2016) geometrically non-linear co-rotational shell FEs have been employed. The detailed FE models have been generated using a versatile mesh-generation computing tool, developed to allow for the plates and core cells to be modelled to any desired level of discretisation detail, as illustrated in Figures 1(a) and 1(b). The adopted strategy has been implemented in ADAPTIC (Izzuddin, 1991), a 

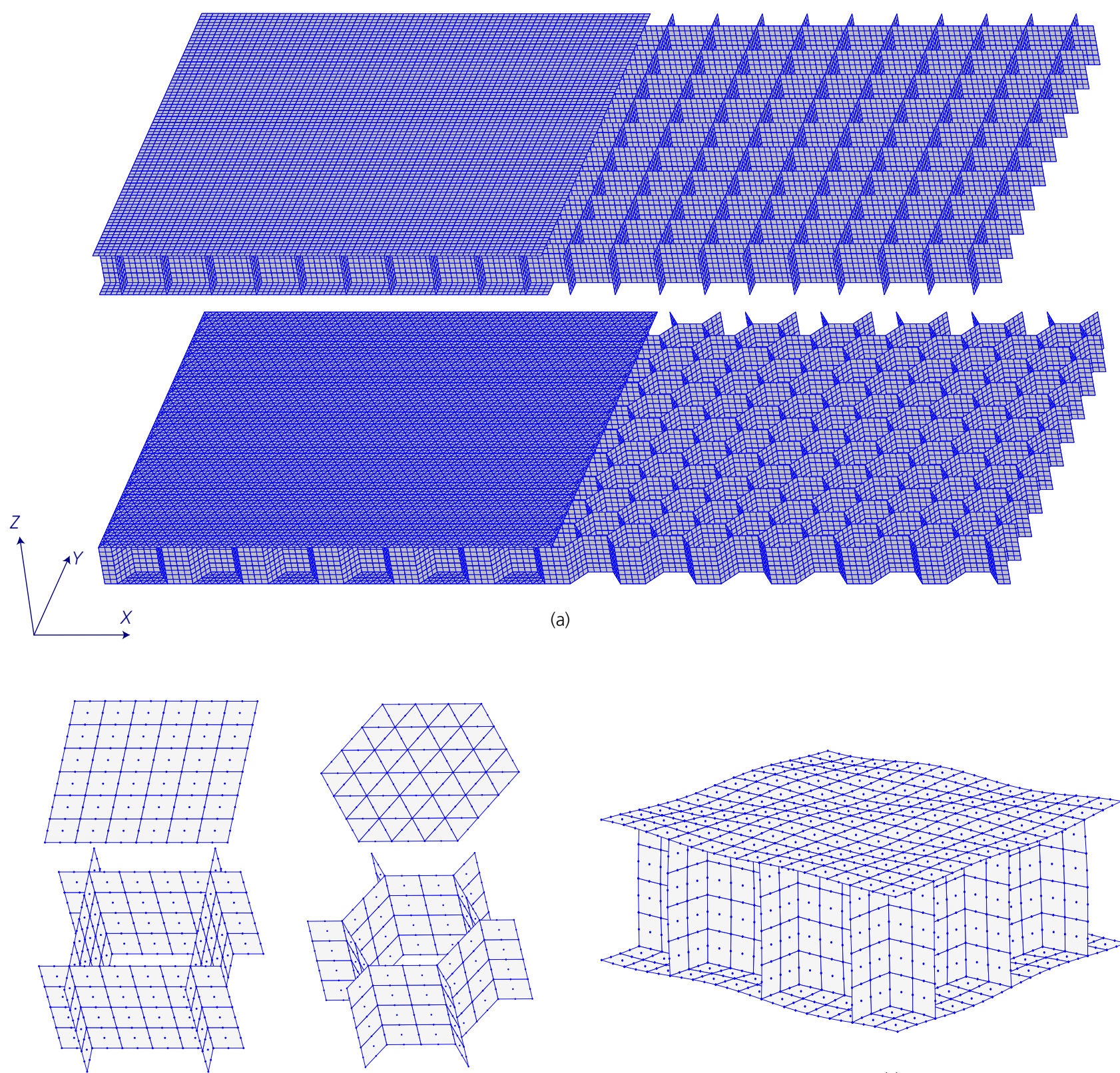

(b)

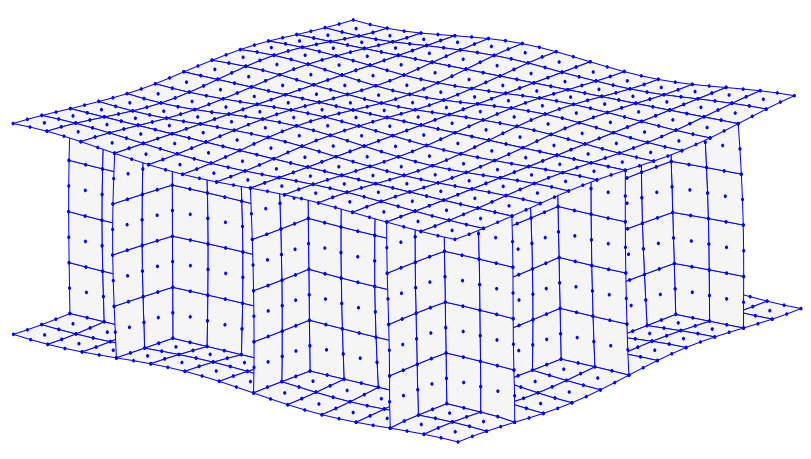

(c)

Figure 1. High-fidelity FE discretisation of rectangular- and hexagonal-honeycomb-core sandwich panels (a), discretisation strategy for rectangular and hexagonal honeycomb cells with nine-noded quadrilateral and six-noded triangular shell FEs (b), sympathetic sinusoidal imperfection modelling idealisation for rectangular-honeycomb-core sandwich panels (c)

sophisticated general FE code for the adaptive non-linear analysis of structures under extreme static and dynamic loading. The principal aspects of the local element formulation and co-rotational transformation are summarised in the following sub-sections for the case of the nine-noded elements, with similar principles also applying in the case of the six-noded elements.

\subsection{Local formulation of hierarchically optimised nine-noded shell FE}

The nine-noded curved shell FE employs five nodal degrees of freedom (DOF) in the local $(x, y, z)$ Cartesian coordinate system, of which three are translational and two rotational. For the interpolation of the local element shape and displacement fields, quadratic Lagrangian shape functions are 
employed in an isoparametric formulation framework, expressed in terms of the natural coordinates $(\xi, \eta)$

1. $\quad \mathbf{x}=\langle x, y, z\rangle^{\mathrm{T}}=\sum_{i=1}^{9} N_{i}(\xi, \eta) \mathbf{x}_{i}$

$$
\boldsymbol{t}=\langle u, v, w\rangle^{\mathrm{T}}=\sum_{i=1}^{9} N_{i}(\xi, \eta) \boldsymbol{t}_{i},
$$

$$
\boldsymbol{r}=\left\langle\theta_{\mathrm{x}}, \theta_{y}\right\rangle^{\mathrm{T}}=\sum_{i=1}^{9} N_{i}(\xi, \eta) \boldsymbol{r}_{i}
$$

where $\mathbf{x}_{i}=\left\langle x_{i}, y_{i}, z_{i}\right\rangle^{\mathrm{T}}$ are the nodal coordinates, and $\boldsymbol{t}_{i}=\left\langle u_{i}, v_{i}\right.$, $\left.w_{i}\right\rangle^{\mathrm{T}}$ and $\boldsymbol{r}_{i}=\left\langle\theta_{i x}, \theta_{i y}\right\rangle^{\mathrm{T}}$ are the translations and rotations of node (i), respectively, in the local coordinate system. The ReissnerMindlin theory underlies the elements' local kinematics, where the employment of a co-rotational approach enables the use of a simplified strain-displacement relationship through the exclusion of rigid-body modes from the local element deformation modes, as will be discussed in more detail in the next section. The membrane, bending and transverse shear element strains can therefore be expressed in terms of the local element displacement fields using the following equations

3.

$$
\varepsilon^{\mathrm{m}}=\left\{\begin{array}{c}
\varepsilon_{x} \\
\varepsilon_{y} \\
\gamma_{x y}
\end{array}\right\}=\left\{\begin{array}{c}
\frac{\partial u}{\partial x} \\
\frac{\partial v}{\partial y} \\
\frac{\partial u}{\partial y}+\frac{\partial v}{\partial x}
\end{array}\right\}
$$

$$
+\left\{\begin{array}{c}
\frac{1}{2}\left(\frac{\partial z}{\partial x}+\frac{\partial w}{\partial x}\right)^{2}-\frac{1}{2}\left(\frac{\partial z}{\partial x}\right)^{2} \\
\frac{1}{2}\left(\frac{\partial z}{\partial y}+\frac{\partial w}{\partial y}\right)^{2}-\frac{1}{2}\left(\frac{\partial z}{\partial y}\right)^{2} \\
\left(\frac{\partial z}{\partial x}+\frac{\partial w}{\partial x}\right)\left(\frac{\partial z}{\partial y}+\frac{\partial w}{\partial y}\right)-\left(\frac{\partial z}{\partial x}\right)\left(\frac{\partial z}{\partial y}\right)
\end{array}\right\}
$$

4. $\boldsymbol{\varepsilon}^{\mathrm{b}}=\left\{\begin{array}{c}\kappa_{x} \\ \kappa_{y} \\ \kappa_{x y}\end{array}\right\}=\left\{\begin{array}{c}\frac{\partial \theta_{x}}{\partial x} \\ \frac{\partial \theta_{y}}{\partial y} \\ \frac{\partial \theta_{x}}{\partial y}+\frac{\partial \theta_{y}}{\partial x}\end{array}\right\}$

5. $\boldsymbol{\varepsilon}^{\mathrm{s}}=\left\{\begin{array}{l}\gamma_{x z} \\ \gamma_{y z}\end{array}\right\}=\left\{\begin{array}{c}\theta_{x}+\frac{\partial w}{\partial x} \\ \theta_{y}+\frac{\partial w}{\partial y}\end{array}\right\}$
The aforementioned kinematic assumptions and conforming compatibility relationships, in conjunction with element shape irregularities, introduce polluting higher-order non-linear terms in the strain distributions. These inaccuracies deteriorate the element performance, leading potentially to shear, membrane and distortion locking, which are the principal locking phenomena encountered in curved shell elements, arising for small shell thickness, curved shapes and the employment of isoparametric mapping for distorted element shapes, respectively. In the adopted formulation, locking phenomena are relieved by correcting each of the conforming membrane, shear and bending strains with corrective hierarchic components $\boldsymbol{\Psi}_{\mathrm{h}}$, towards an objective set of strains $\boldsymbol{\Psi}_{\mathrm{o}}$ afforded by the original DOFs, using appropriate parameters (Izzuddin and Liang, 2017)

6. $\varepsilon+\varepsilon_{\mathrm{h}} \rightarrow \varepsilon_{\mathrm{o}}, \quad \varepsilon_{\mathrm{h}}=\boldsymbol{\Psi}_{\mathrm{h}} \boldsymbol{\alpha}_{\mathrm{h}}, \quad \varepsilon_{\mathrm{o}}=\boldsymbol{\Psi}_{\mathrm{o}} \boldsymbol{\alpha}_{\mathrm{o}}$

The hierarchic and objective parameters, $\boldsymbol{\alpha}_{\mathrm{h}}$ and $\boldsymbol{\alpha}_{\mathrm{o}}$, are determined by minimising the error between the corrected and objective strains, which yields

7. $\quad\left(\int_{\Omega^{e}}\left[\begin{array}{c|c}\boldsymbol{\Psi}_{\mathrm{h}}^{\mathrm{T}} \boldsymbol{\Psi}_{\mathrm{h}} & -\boldsymbol{\Psi}_{\mathrm{h}}^{\mathrm{T}} \boldsymbol{\Psi}_{\mathrm{o}} \\ \hline-\boldsymbol{\Psi}_{\mathrm{o}}^{\mathrm{T}} \boldsymbol{\Psi}_{\mathrm{h}} & \boldsymbol{\Psi}_{\mathrm{o}}^{\mathrm{T}} \boldsymbol{\Psi}_{\mathrm{o}}\end{array}\right] \mathrm{d} \Omega^{e}\right)\left\{\frac{\boldsymbol{\alpha}_{\mathrm{h}}}{\boldsymbol{\alpha}_{\mathrm{o}}}\right\}=\int_{\Omega^{e}}\left[\begin{array}{c}-\boldsymbol{\Psi}_{\mathrm{h}}^{\mathrm{T}} \\ \hline \boldsymbol{\Psi}_{\mathrm{o}}^{\mathrm{T}}\end{array}\right] \boldsymbol{d} \mathrm{d} \Omega^{e}$

The hierarchic optimisation approach leads to a family of nine-noded elements, with two alternative branches $\mathrm{H} n \mathrm{O} 9$ and $\mathrm{H} n \mathrm{C} 9$, where $n$ indicates the order of hierarchic modes and $\mathrm{O} \mid \mathrm{C}$ refers to the employment of objective or corrected strains to substitute the conforming field. The $\mathrm{H} 3 \mathrm{O} 9$ variant has been shown to achieve optimal performance in addressing all three forms of locking (Izzuddin and Liang, 2017) and is therefore employed herein.

\subsection{Co-rotational transformations}

The co-rotational approach encompasses the definition of a local coordinate system which follows the element in its deformed configuration, thus excluding rigid-body translations and importantly rotations, thus enabling the use of simplified kinematic relationships at the local element level. For the optimised nine-noded $\mathrm{H} 3 \mathrm{O} 9$ shell $\mathrm{FE}$, a simple definition of the local $x$ - and $y$-axis as the bi-sectors of the diagonals fulfils the requirements for an effective co-rotational approach, specifically (i) simplified definition, (ii) nodal invariance, (iii) element spin reduction and (iv) uniqueness in the local system definition, resulting in symmetric tangent stiffness matrix (Izzuddin and Liang, 2016). As illustrated in Figure 2, the relative orientation of the local co-rotational system in the element 


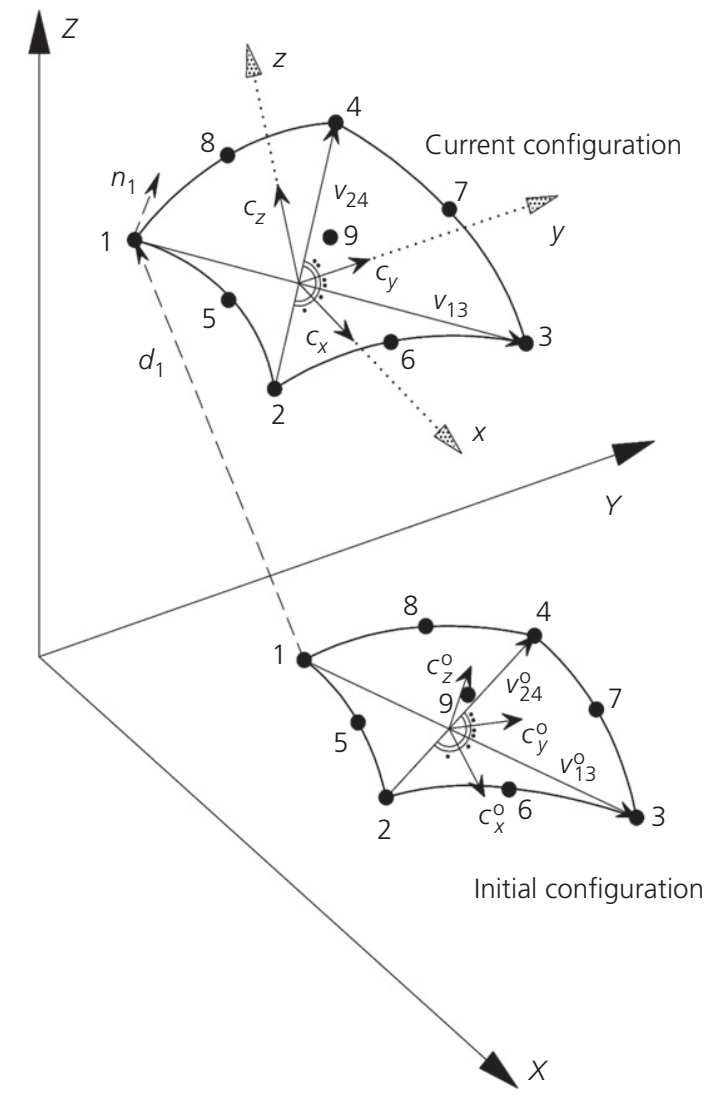

Figure 2. Bisector local co-rotational system and global nodal displacement parameters for nine-noded quadrilateral shell FE (Izzuddin and Liang, 2017)

deformed configuration with reference to the global system is uniquely defined by the orthogonal vector basis $\left(\boldsymbol{c}_{x}, \boldsymbol{c}_{y}, \boldsymbol{c}_{z}\right)$, where the triad is obtained as

8. $\quad \boldsymbol{c}_{x}=\frac{\boldsymbol{c}_{13}-\boldsymbol{c}_{24}}{\left|\boldsymbol{c}_{13}-\boldsymbol{c}_{24}\right|}, \quad \boldsymbol{c}_{y}=\frac{\boldsymbol{c}_{13}+\boldsymbol{c}_{24}}{\left|\boldsymbol{c}_{13}+\boldsymbol{c}_{24}\right|}, \quad \boldsymbol{c}_{z}=\boldsymbol{c}_{x} \times \boldsymbol{c}_{y}$

with

9. $\quad c_{i j}=\frac{v_{i j}}{\left|v_{i j}\right|}, \quad v_{i j}=v_{i j}^{(0)}+d_{j}-d_{i}$

In the above expressions, $v_{i j}^{(0)}$ and $v_{i j}$ are the vectors linking nodes $(i)$ and $(j)$ in the initial undeformed and the current deformed configurations, respectively, and $\boldsymbol{d}_{i}=\left\langle U_{i}, V_{i}, W_{i}\right\rangle^{\mathrm{T}}$ is the global translation vector of node $(i)$.

The co-rotational approach allows for the local internal force vectors and tangent stiffness matrices to be obtained from local deformations that exclude element rigid-body modes, while the geometric non-linearity associated with the latter is incorporated through relevant discrete transformations between the global and local coordinate systems. For the translational and rotational DOFs, these transformations can be expressed as

10. $\boldsymbol{t}_{i}=\mathbf{R} \boldsymbol{d}_{i}+\left(\mathbf{R}-\mathbf{R}^{\mathrm{o}}\right) \boldsymbol{v}_{i \mathrm{o}}^{\mathrm{o}}$

\section{1. $\quad \overline{\boldsymbol{r}}_{i}=-\overline{\mathbf{R}} \boldsymbol{n}_{i}$}

where $\boldsymbol{n}_{i}$ is the normal vector and $\boldsymbol{v}_{i \mathrm{o}}^{\mathrm{o}}$ the initial position vector about the diagonal intersection, of node $(i)$, while $\mathbf{R}^{\circ}$ and $\mathbf{R}, \overline{\mathbf{R}}$ are the orientation matrices of the co-rotational system in the initial and deformed element configurations

12.

$$
\begin{aligned}
& \mathbf{R}^{\mathrm{o}}=\left[\begin{array}{lll}
\boldsymbol{c}_{x}^{\mathrm{o}} & \boldsymbol{c}_{y}^{\mathrm{o}} & \boldsymbol{c}_{z}^{\mathrm{o}}
\end{array}\right]^{\mathrm{T}}, \quad \mathbf{R}=\left[\begin{array}{lll}
\boldsymbol{c}_{x} & \boldsymbol{c}_{y} & \boldsymbol{c}_{z}
\end{array}\right]^{\mathrm{T}}, \\
& \overline{\mathbf{R}}=\left[\begin{array}{ll}
\boldsymbol{c}_{x} & \boldsymbol{c}_{y}
\end{array}\right]^{\mathrm{T}}
\end{aligned}
$$

Two additional transformations are required to transform the local element resistance forces and tangent stiffness matrix from the local co-rotational system to the global system, which are obtained considering the first- and second-order derivatives of the above geometric relationships linking the local to global DOF parameters (Izzuddin and Liang, 2016).

It is worth noting that the orientation of the nodal normal $\boldsymbol{n}_{i}$ at node $(i)$ is determined by three global rotational DOFs, but obviously the 'drilling' rotation will have no influence on the orientation of $\boldsymbol{n}_{\boldsymbol{i}}$. This means that the element exhibits a singular behaviour in relation to the drilling DOF, which must either be excluded or restrained when discretising a smooth shell surface. When it comes to the modelling of complex shell structures, including parts with smooth surfaces and other parts intersecting at an angle, the most effective approach would be to utilise three global rotational DOFs per node, and restrain the drilling DOF, accounting for the changing orientation of the drilling normal vector in large displacement analysis. This has implications on the modelling of all-metal sandwich panels, where careful consideration should be given to the treatment of drilling rotational DOFs, particularly along a partitioned boundary with domain decomposition, as discussed in Section 3.2.

\section{Partitioned modelling of large-scale metal sandwich panels}

In non-linear static or dynamic analysis problems where FE procedures are employed, the computational demand is inextricably related to the numerical solution of the algebraic 
system of equations of discrete equilibrium for a given set of essential and natural boundary conditions. The size of this system of equations is determined by the number of DOF parameters employed for the domain discretisation, thus increasing substantially in the case of dense or large-scale meshes. For a detailed non-linear response evaluation of large-scale metal sandwich panels in accordance with the highfidelity modelling strategy discussed in Section 2, several hundreds of thousands of DOFs are typically required for a realistic idealisation, rendering the simulation prohibitively expensive, even impossible, in relation to computing time and memory resources. These shortcomings are overcome in the proposed modelling strategy through the application of a recently developed partitioned modelling approach (Jokhio and Izzuddin, 2015), as elaborated in the following sections.

\subsection{Dual super-element partitioned modelling}

A highly efficient domain decomposition approach for parallel processing is employed in this work to enhance the computational efficiency and to address the memory bottlenecks of conventional non-linear analysis. This approach utilises the novel dual super-element concept (Jokhio and Izzuddin, 2015), which enables the straightforward upgrade of existing nonlinear FE programmes to scalable parallel processing over computing systems with distributed memory. The method involves the domain decomposition of an initial parent structure into place-holder super-elements along the partition boundary, each representing a child partition. Place-holder superelements are treated as conventional FEs in the parent process, and these communicate with dual super-elements that wrap the child partition which is analysed in a separate process. At every iterative step of the solution procedure, the displacements at the partition boundary nodes are communicated from the placeholder to the dual super-elements, and subsequently the condensed resistance forces vector and tangent stiffness matrix for each dual super-element are obtained by way of a backward substitution and forward elimination processes at the level of the child partition, after which they are communicated back to the placeholder super-element in the parent process (Jokhio and Izzuddin, 2015). This two-way communication between pairs of placeholder and dual super-elements allows the discrete conditions of compatibility and equilibrium to be enforced within the individual partitions, including the parent partition, and along the partition boundary, while eliminating the requirement for communication between child partitions.

The development of the methodology to allow for scalable parallel processing enables its direct implementation in a highperformance computing (HPC) system, where individual child and parent processes are allocated to independent central processing units (CPU), or cores, distributed over single or multiple computing nodes. The reduced number of solver operations within separate child processes and the effective parallelisation of the solution procedure led to a remarkable speed-up and reduction in the computing memory demand in comparison with the respective monolithic models. Furthermore, domain partitioning achieves identical results and convergence rate to the monolithic approach, while allowing for memory bottlenecks to be effectively overcome.

An advanced high-fidelity mesh-generation tool for large-scale metal sandwich panels has been developed to facilitate domain partitioning in ADAPTIC (Izzuddin, 1991), which incorporates an implementation of the above dual super-element partitioning approach. This allows the domain decomposition of sandwich panel FE models into child partitions, each of which is in turn discretised with the geometrically non-linear co-rotational shell FEs discussed in Section 2. A schematic representation of the concept is presented in Figure 3, where the partition boundary comprises only the nodes along the individual child partition domain perimeters.

\subsection{Drilling DOF constraints on partitioned boundary}

As discussed in Section 2, the adopted shell elements utilise six DOFs, with three rotational DOFs, per node, although the local element response is formulated with five local DOFs, with two rotational DOFs, for each node. This is typical of shell elements utilising the basic nodal parameters and makes these elements insensitive to 'drilling' nodal rotations, thus exhibiting a singular response under such rotations. Such singularity does not arise for nodes along edges where elements intersect at an angle, such as for a folded plate or at the intersection of the honeycomb core with the faceplates for an all-metal sandwich panel. Accordingly, the most general approach would be to utilise three rotational DOFs per node, and to constrain the drilling DOF at nodes where there are no intersections at an angle between adjacent shell elements (i.e. locations where the discretised domain is a smooth surface with no intersections). This is indeed the approach considered in ADAPTIC (Izzuddin, 1991) for the utilised six- and ninenoded shell elements, where drilling DOF constraints are automatically introduced at non-intersecting nodal locations, achieved by way of a special constraint element that allows for the variation of the drilling orientation vector with large displacements.

In the context of partitioned modelling, however, the automatic introduction of the drilling constraint element is restricted to the internal nodes of the partition. This is because the partitions are processed as substructures on separate processors, and it is not possible without more advanced communication between child processes to establish whether the nodes on a partitioned boundary belong to a smooth 


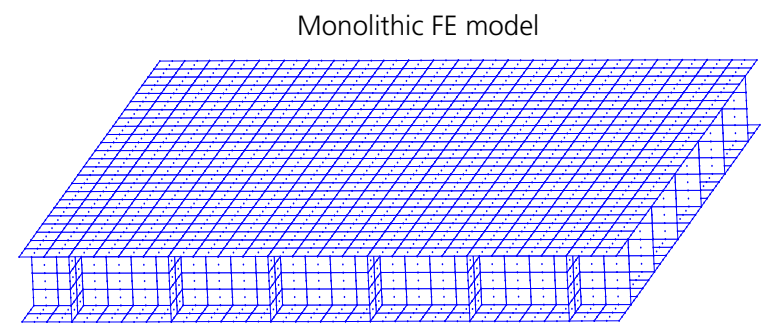

Domain decomposition - partitioned

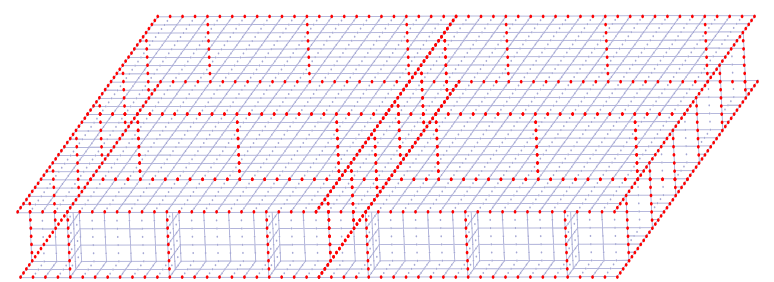

Level (0): partition boundary and placeholder super-elements

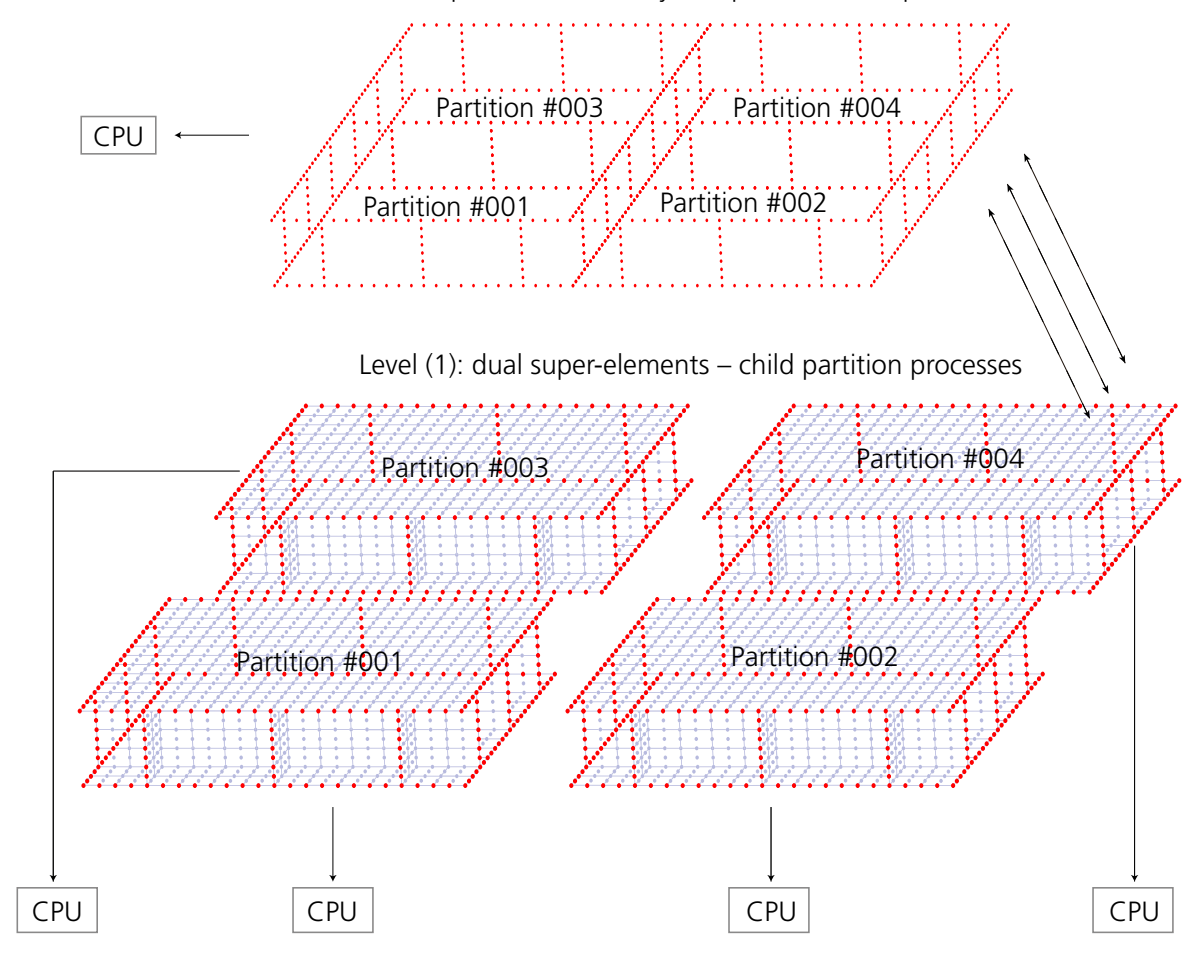

Figure 3. Domain decomposition of rectangular-honeycomb-core sandwich panels using dual super-element child partitions

part of the domain or to an intersection between two or more surfaces. Therefore, to address the singularity associated with the drilling DOF on a partitioned boundary where there are no intersections, additional constraint elements are introduced at the level of the parent partitions, as illustrated in Figure 4. This approach has been incorporated in the developed mesh generator for all-metal sandwich panels, so that the additional drilling DOF constraint elements are introduced automatically for effective partitioned modelling of such panels.

\section{Application studies}

In this section, the proposed high-fidelity modelling strategy is employed for investigating the non-linear response and characterising the failure modes of large-scale metal sandwich panels 


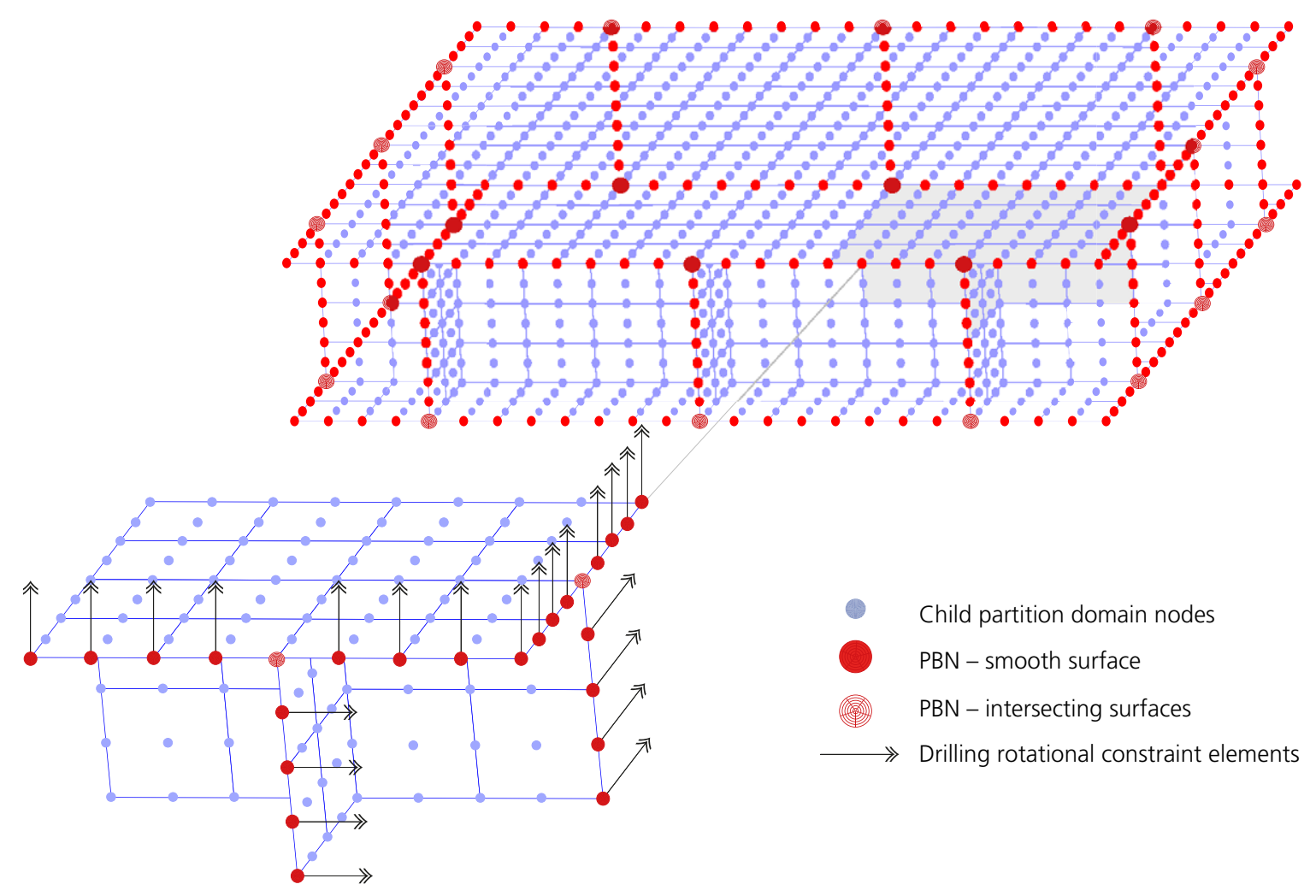

Figure 4. Drilling rotational constraint elements along smooth part of partition boundary

with rectangular $(R)$ and hexagonal $(H)$ honeycomb core topologies, further highlighting its capability of accurately capturing associated complex phenomena. The benefits of the adopted partitioned modelling approach are also emphasised with respect to reducing the computational wall-clock time and overcoming potential memory bottlenecks. A comparative evaluation of the efficiency of partitioned models with different numbers of child partitions, comparing the achieved speed-up relative to equivalent monolithic models, is finally presented, where guidance is provided for selecting the optimal number of child partitions.

For the purpose of the presented numerical studies, consideration is given as base cases to large-scale $3000 \times 3000 \mathrm{~mm}^{2}$ sandwich panels, with $100 \mathrm{~mm}$ depth between the mid-planes of the top and bottom plates, and a $3 \mathrm{~mm}$ thickness for the top and bottom plates and the core cell walls. Regular cell geometries have been adopted, with $90^{\circ}$ and $120^{\circ}$ cell wall angles for the rectangular and hexagonal honeycomb cores, respectively. The rectangular cell dimensions along the orthogonal global planar $X$ - and $Y$-axis have been set to $100 \times 100 \mathrm{~mm}^{2}$, while for the hexagonal cells an equivalent core density is obtained for a cell wall width of $60 \mathrm{~mm}$, leading to
$120 \times 104 \mathrm{~mm}^{2}$ cells. The connections at the core cell intersections, as well as between the core and the plates, are considered to be fully rigid. A triaxial elastoplastic constitutive model is adopted, with a yield strength $f_{\mathrm{y}}=355 \mathrm{~N} / \mathrm{mm}^{2}$, Young's modulus $E=210000 \mathrm{~N} / \mathrm{mm}^{2}$ and Poisson's ratio $v=0 \cdot 3$, where the influence of strain hardening is neglected. All panels have been modelled as simply supported along all four edges, with translational restraints introduced at the nodes along the bottom plate perimeter is the global $Z$ directions, and minimal planar restraint is provided to prevent rigid-body motion.

\subsection{Characteristic non-linear response of large-scale sandwich panels}

Particular focus is given herein to two-way spanning largescale deck system applications, where sandwich panels are employed as primary structural components, considering uniformly distributed loading (UDL) and patch loading (PL) as the main load cases. The loads are applied in proportion to a load factor, with nominal values of $p_{\text {nom }}=260 \mathrm{kN} / \mathrm{m}^{2}$ and $P_{\text {nom }}=800 \mathrm{kN}$ for the UDL and PL cases, respectively, where the latter is applied over a stiff, practically rigid, $300 \times 300 \mathrm{~mm}^{2}$ baseplate. The terminating condition for all 
analyses is set to a maximum value of global transverse displacement of $300 \mathrm{~mm}$, corresponding to $10 \%$ of the global panel dimensions, beyond which excessive strains of the order of the ultimate strain for mild steel are typically achieved. For the purpose of establishing imperfection sensitivity, alternative sympathetic sinusoidal imperfections are introduced in the rectangular-honeycomb-core models, with respective amplitudes of $0 \cdot 1,0 \cdot 2,0 \cdot 5,1 \cdot 0$ and $2 \cdot 0 \%$ of the minimum plated component dimension, where zero displacements and compatible rotations along the boundaries are considered, as illustrated in Figure 1(c).

The non-linear equilibrium path for the vertical displacement of the bottom plate central node is presented in Figures 5(a) and 5(b) for the UDL and PL cases, respectively, for two different mesh densities employing 43200 and 230400 elements, henceforth denoted as conventional (C) and high fidelity (HF), for both rectangular (R-C, R-HF) and

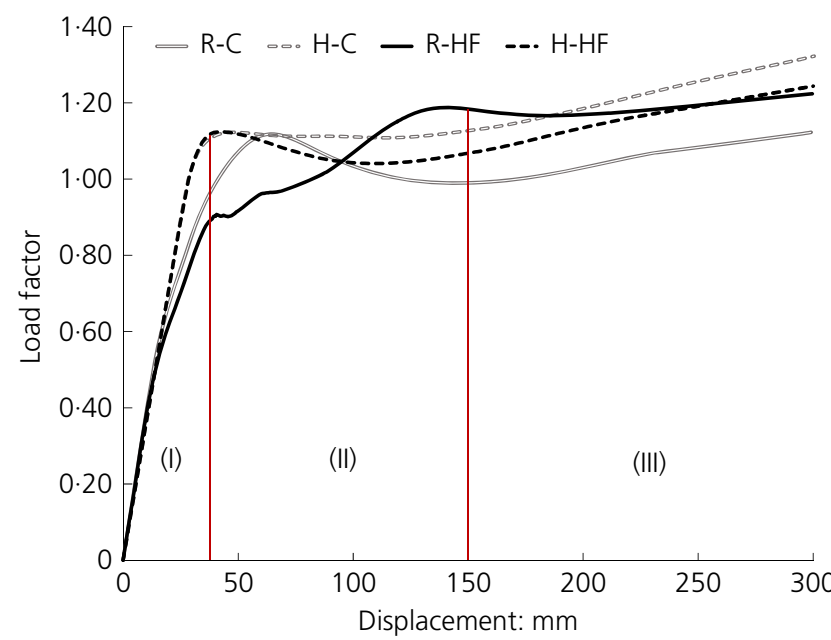

(a)

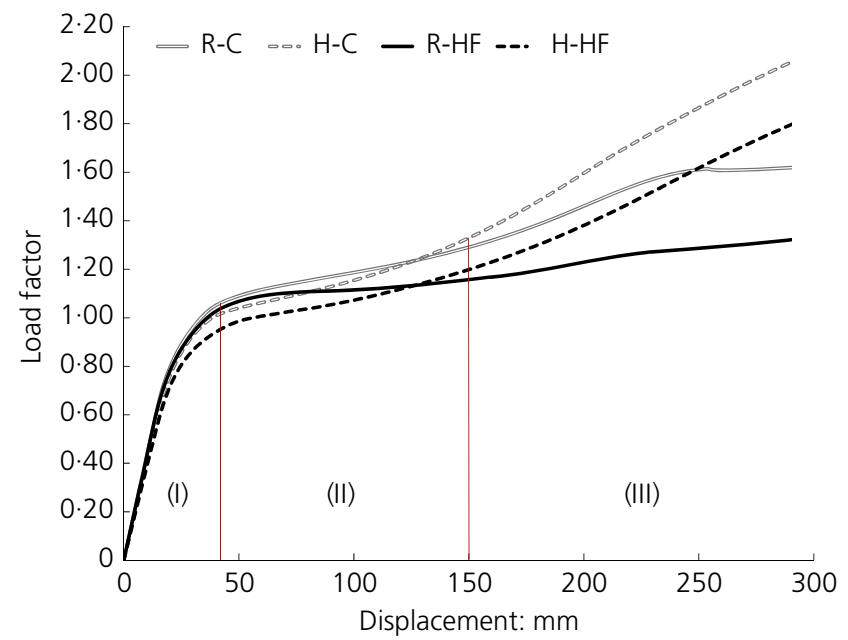

(b)

Figure 5. Non-linear response of sandwich panels subject to UDL (a) and PL (b)

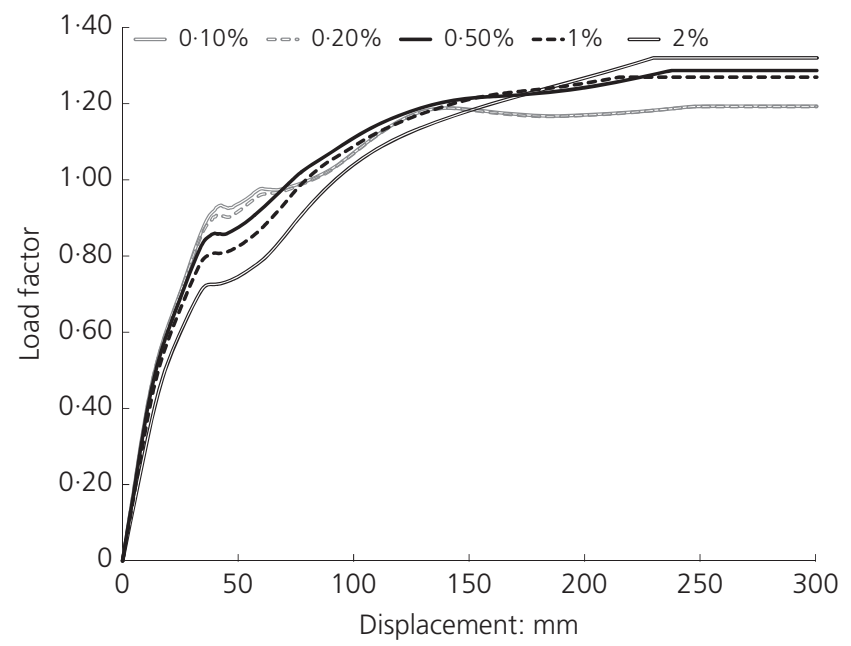

(a)

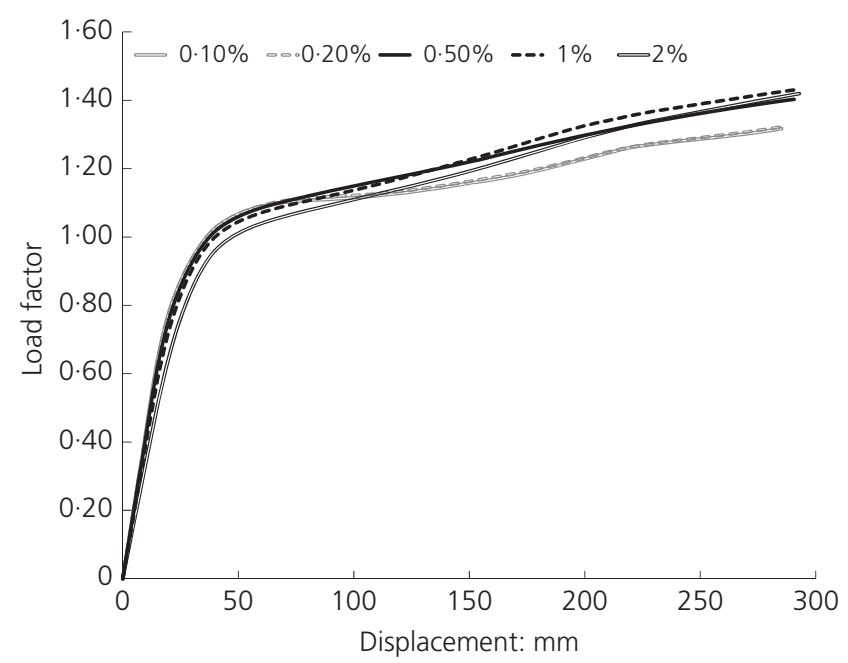

(b)

Figure 6. Imperfection sensitivity of rectangular-honeycomb-core sandwich panels to UDL (a) and PL (b) 
High-fidelity non-linear analysis of metal

sandwich panels

Nordas, Santos, Izzuddin and Macorini hexagonal (H-C, H-HF) core topologies. The results of the imperfection sensitivity analysis for the R-HF models are illustrated in Figures 6(a) and 6(b) for the UDL and PL cases. The substantial response sensitivity to the employed level of discretisation detail, as well as to the magnitude of imperfections, highlights the necessity of accurately capturing the influence of local plate buckling, including material non-linearity, on the panel response, as it profoundly affects the global response. The proposed HF modelling approach enables the capturing of the stiffness and strength reduction for the rectangular honeycomb panel between the conventional (R-C) and high-fidelity (R-HF) models, while the conventional (H-C) model evidently fails to capture the substantial post-yield softening instability exhibited by the high-fidelity (H-HF) models. The complexities observed in the non-linear response can be attributed to the manifestation of elastoplastic local buckling in several locations over the panel domain, which can only be captured when an extensive level of discretisation detail is employed. Although seemingly unimportant for large-scale systems, imperfections can significantly alter the global non-linear response, due to the latter being governed by local instability phenomena in the large-displacement range. This is particularly crucial for the energy dissipation capability and robustness assessment of individual components and deck systems, where extreme static and dynamic loading can be important design and assessment considerations.

The deformed shape for both configurations in ultimate displacement conditions, alongside local contour plots of the bending moment generalised stress entity along the local
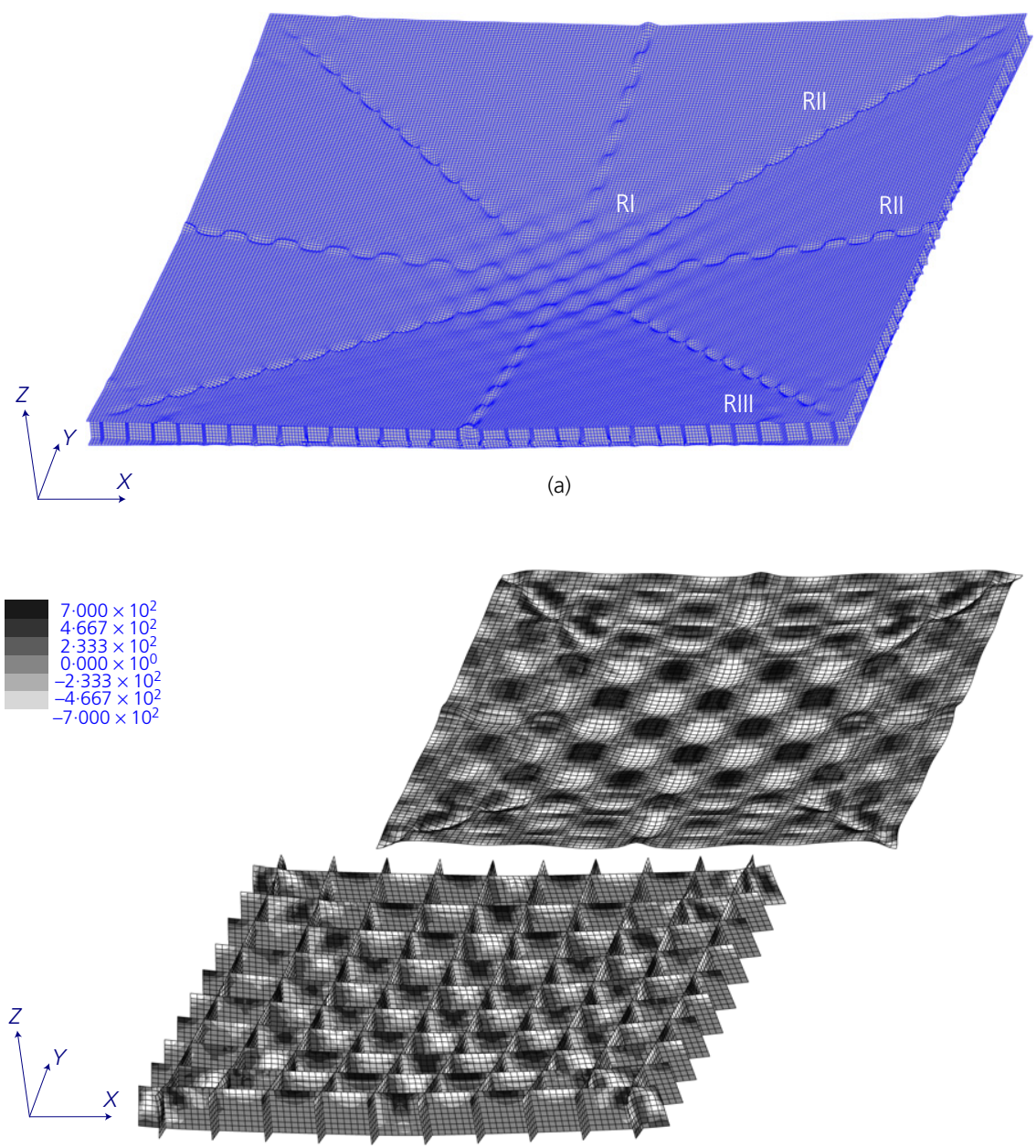

(b)

Figure 7. Deformed configuration at maximum displacement (a) and contours of local $y$-axis bending moment (b-d) for rectangular honeycomb configurations subject to UDL (continued on next page) 


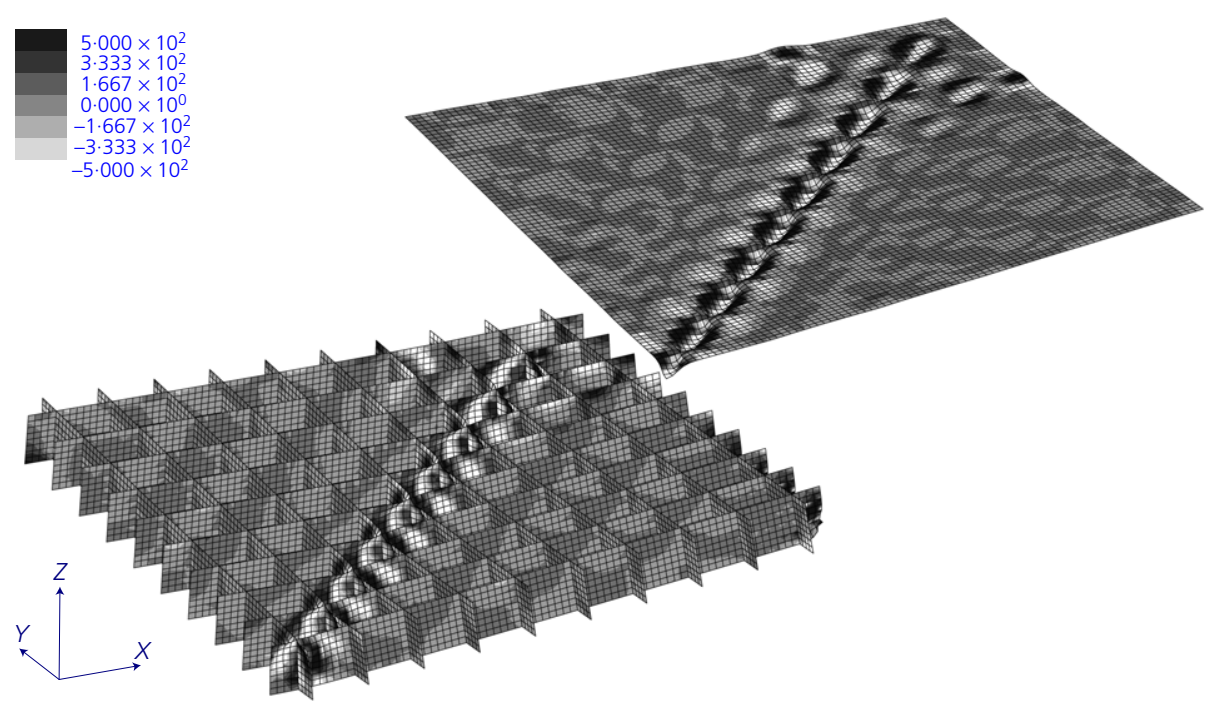

(c)

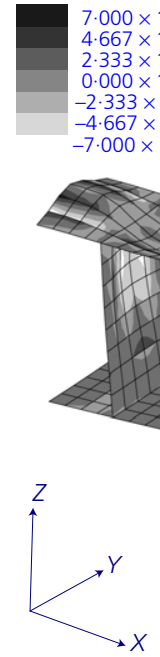

$000 \times 10^{2}$

$4.667 \times 10^{2}$

$0.000 \times 10^{0}$

$.333 \times 10^{2}$

$-7.000 \times 10^{2}$

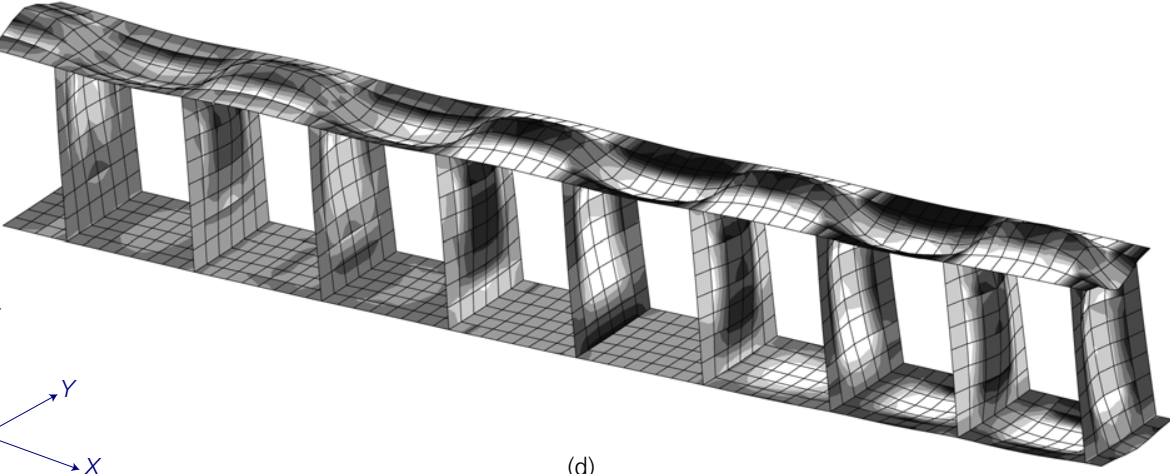

(d)

Figure 7. Continued

element $y$-axis, projected along the global $Y$-axis, at the regions where local instabilities are manifested, are illustrated in Figures 7-10.

Considering more closely the non-linear response under UDL in Figure 5(a), the elastic flexural response of both rectangular and hexagonal honeycomb configurations in region $\mathrm{I}$ is followed by yielding of both plates at the central region, along with elastoplastic intercellular buckling of the top faceplate between the cell walls (Figures 7(b) and 9(b)). Region II is associated with yield line formation, extending towards the four panel corners in the direction of maximum shear stresses at $45^{\circ}$ epicentral angles (Figures 7(c) and 9(c)). The yield line propagation induces primarily shear yielding and subsequent plastic shear buckling of the plate on top of the intersected cells, which is further accompanied by the formation of lines of buckled cells along the horizontal and vertical planar axes in the R-HF case (Figure 7(d)). In region III related to large displacements, membrane action results in response stiffening, while perimetric hoop action induces buckling of a number of half-cells in R-HF.

On the other hand, considering the non-linear response under PL in Figure 5(b), the elastic response of region I is characterised by global flexural deformation of the panel and localised shear deformation of the core underneath the load patch, where the cells are subjected to substantial shear and compression. Shear yielding at the patch perimeter in region II is 

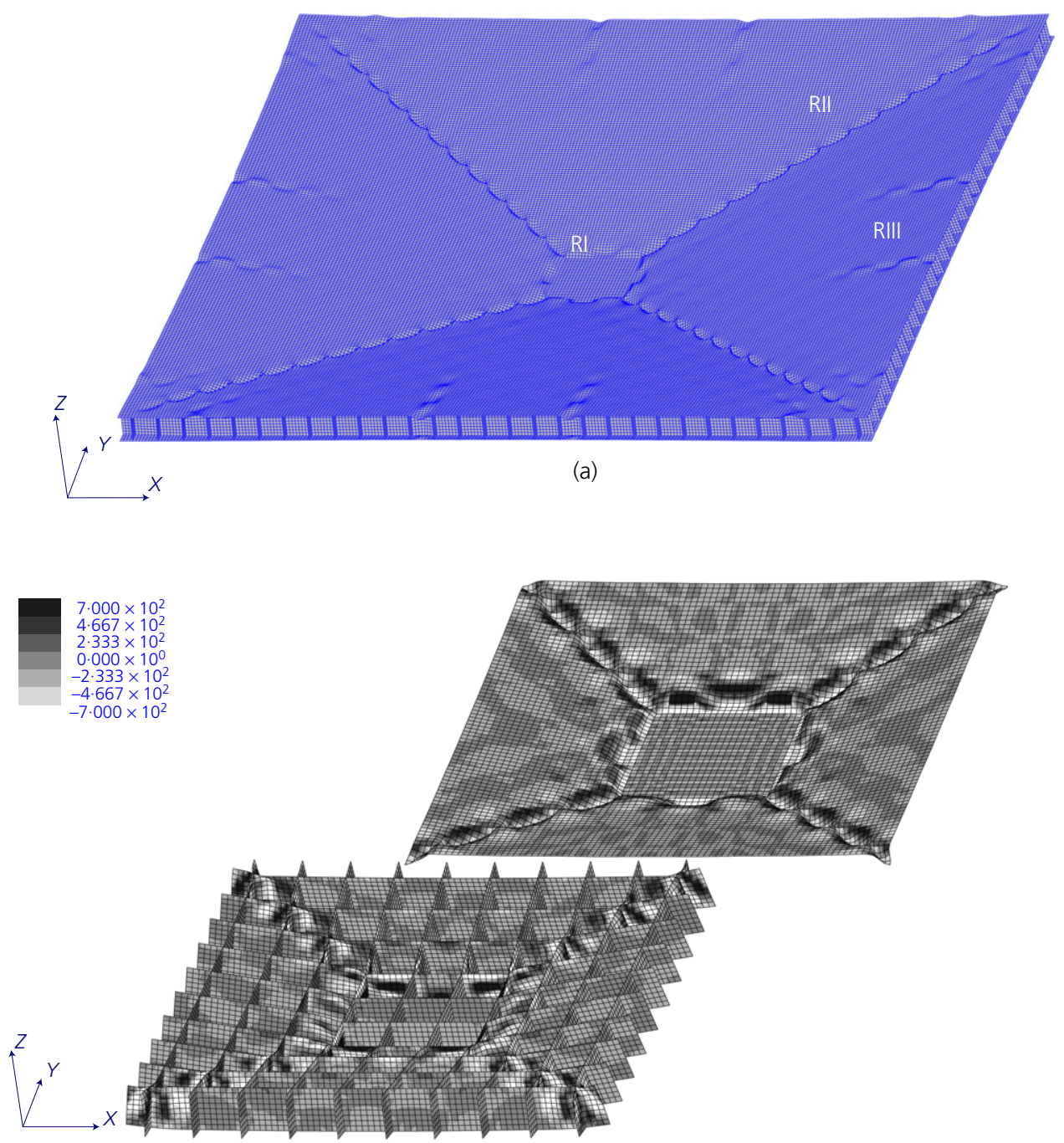

(b)

Figure 8. Deformed configuration at maximum displacement (a) and contours of local $y$-axis bending moment (b-d) for rectangular honeycomb configurations subject to PL (continued on next page)

followed by penetration of the top plate and compressive buckling of the underlying cells, along with shear buckling of the top plate between the cell walls in the patch vicinity (Figures 8(b), 8(d), 10(b), 10(c)). Plasticity spreads in a regular pattern by way of yield line formation and expansion to the four panel corners for R-HF, while a more dispersive pattern is observed for H-HF (Figures 8(c) and 10(b)). Similar to the UDL case, excessive displacements in region III induce perimetric hoop action, which ultimately leads to buckling of a number of half-cells over the panel perimeter in R-HF.

Clearly, such complex response characteristics and localised deformations, can only be captured with high-fidelity modelling. However, this type of modelling is generally associated with high computational and memory demands that can become prohibitive for large-scale application, particularly where multiple interacting panels are considered. The following section demonstrates the benefits of partitioned modelling towards addressing these shortcomings of the conventional modelling approach.

\subsection{Computational benefits of partitioned modelling}

Wall-clock time is employed here to quantify the efficiency of different partitioned modelling configurations, with identical memory resources allocated to individual processes associated with the various partitions. The wall-clock time encompasses the CPU time related to the determination of the non-linear element 


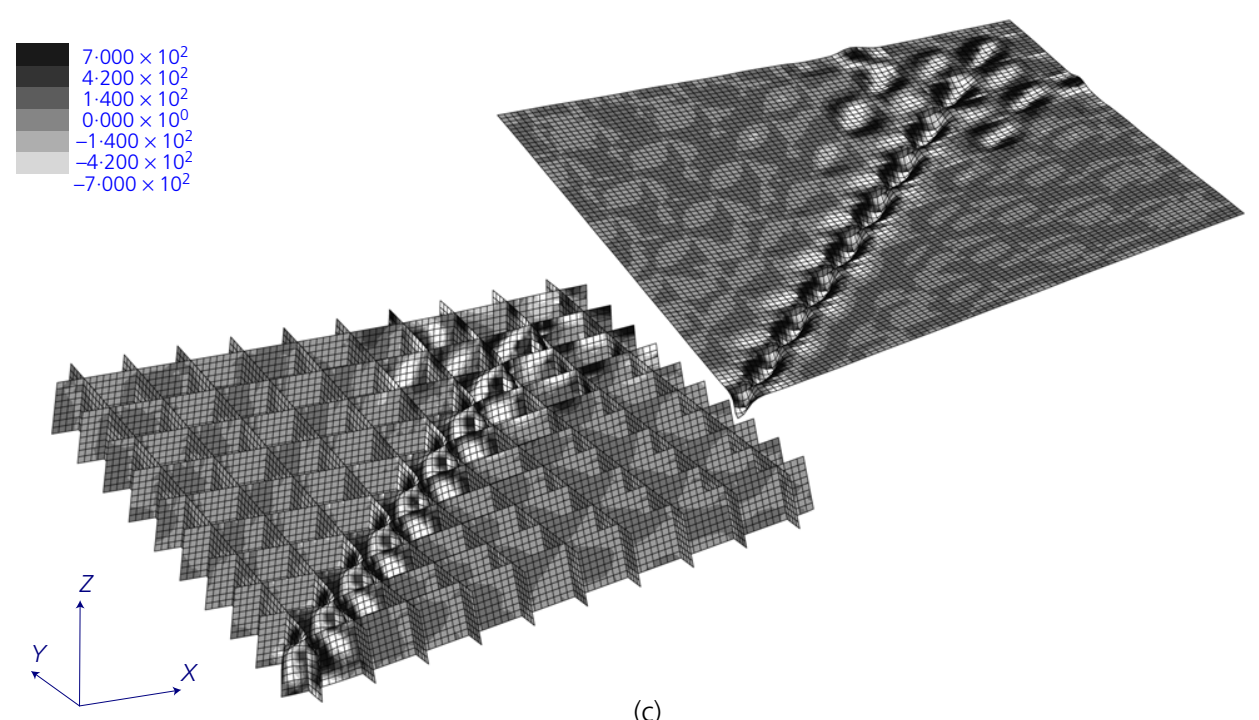

(c)
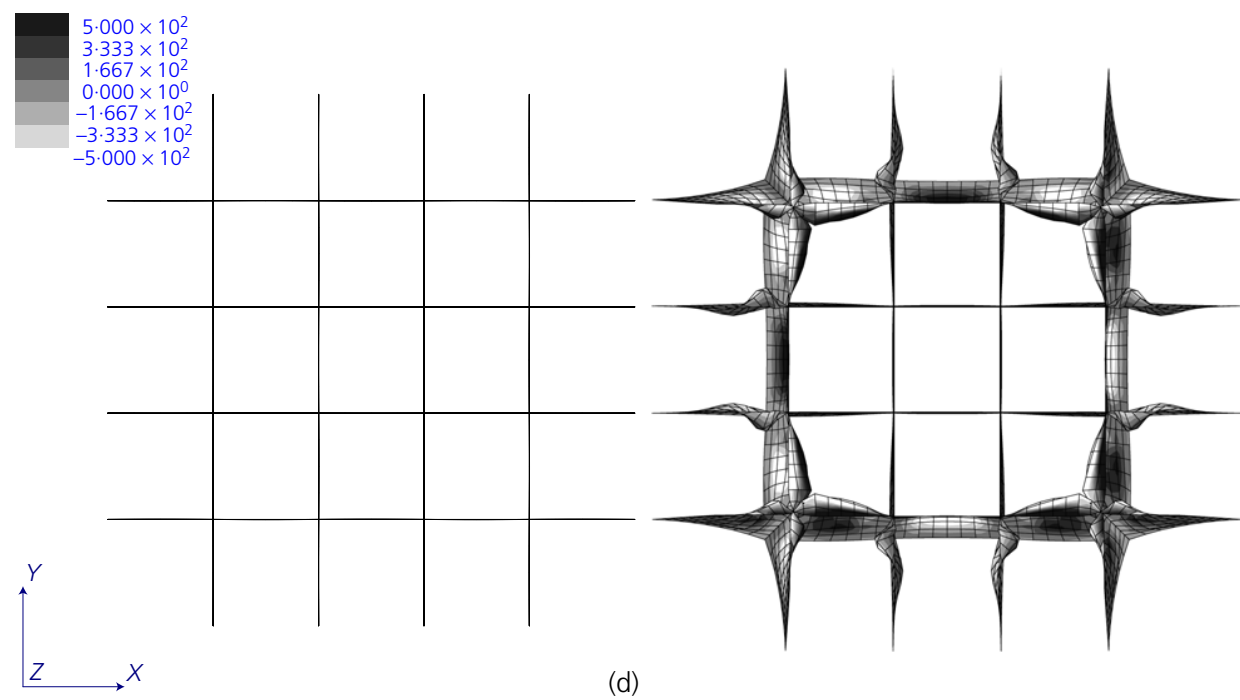

(d)

Figure 8. Continued

response and the numerical solution of the algebraic system of non-linear equations, along with the communication overhead between different processors. Considering the geometric symmetry and mesh uniformity, configurations with two (PM-4), three (PM-9), four (PM-16), five (PM-25), six (PM-36), seven (PM-49) and eight (PM-64) child partitions along the global $X$ and $Y$ axes are considered, resulting in a similar number of DOFs per child process. All analyses are processed using the HPC facility of Imperial College London, where computing nodes with identical processor specifications, including $128 \mathrm{~GB}$ memory, are used in a fully dedicated mode to minimise the inter-processor communication overhead. Two levels of discretisation are considered in base models employing 43200 (BM-1) and 230400 (BM-2) elements, corresponding to R-C and R-HF subjected to UDL, in order for the relative efficiency of domain partitioning using dual super-elements for a moderate and high level of discretisation detail to be investigated.

The speed-up factor $\mathrm{SF}_{p}$ associated with a partitioned model with $p$ child partitions is defined as

13. $\mathrm{SF}_{p}=\frac{t_{\mathrm{mono}}}{t_{p}}$ 
High-fidelity non-linear analysis of metal

sandwich panels

Nordas, Santos, Izzuddin and Macorini
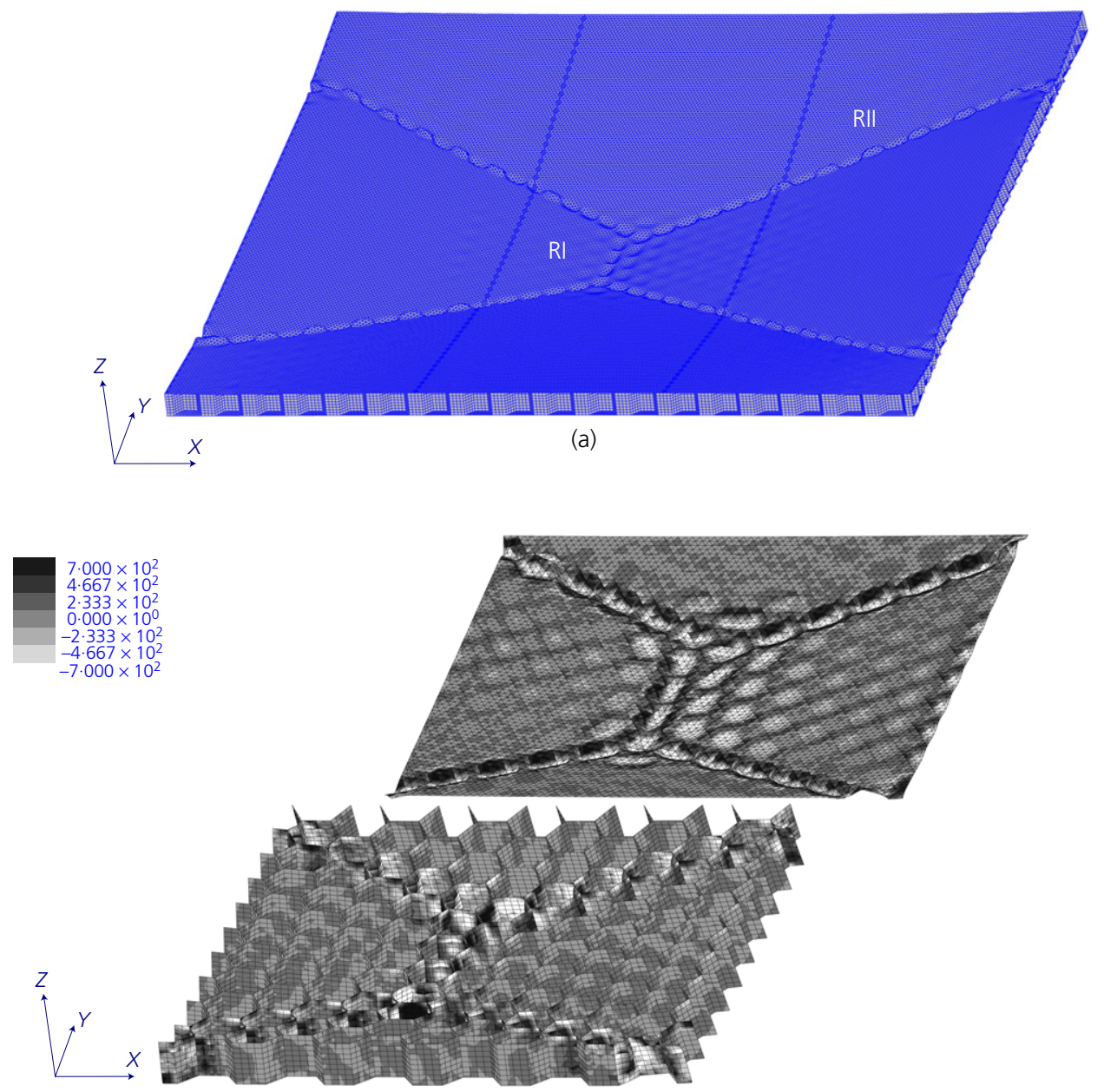

(b)

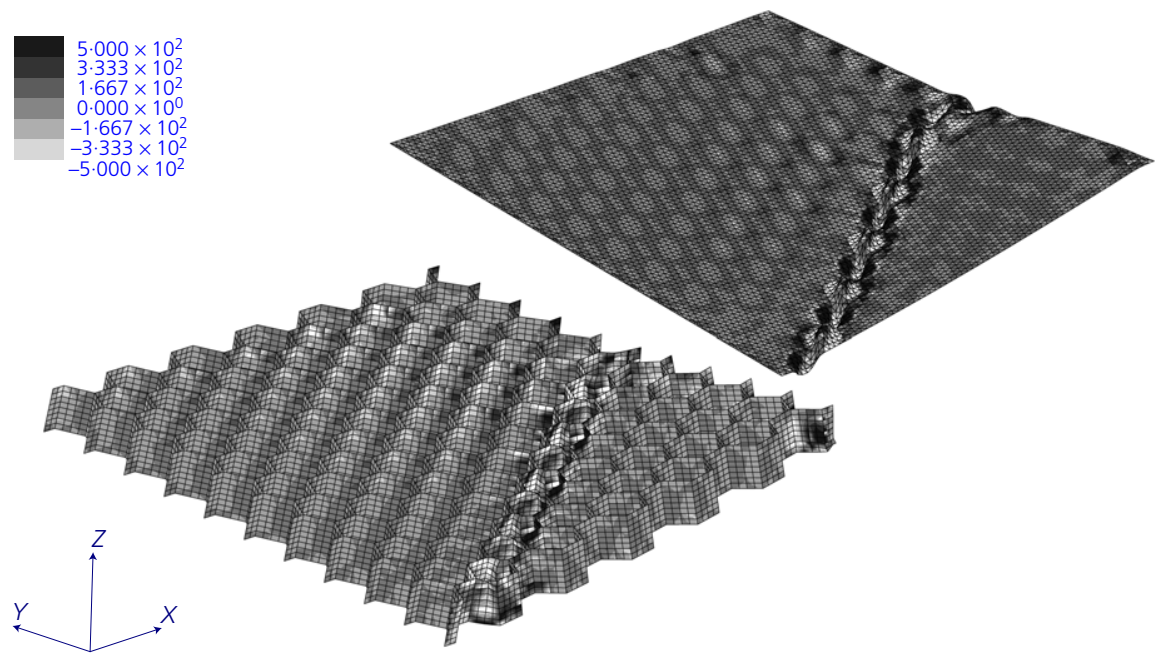

(c)

Figure 9. Deformed configuration at maximum displacement (a) and contours of local $y$-axis bending moment (b, c) for hexagonal honeycomb configurations subject to UDL 


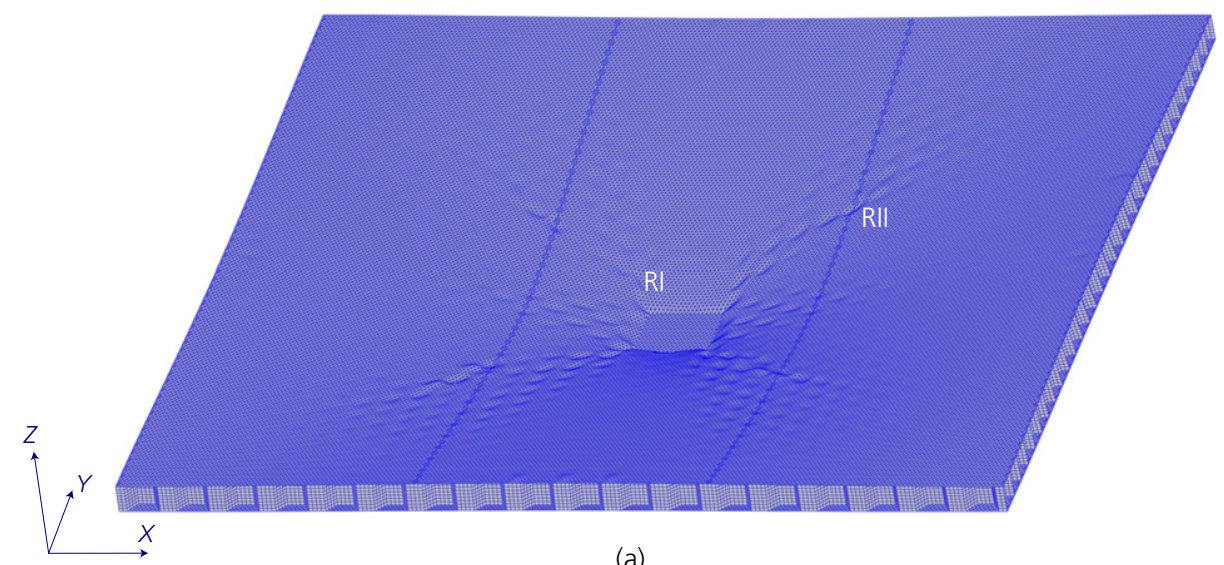

(a)

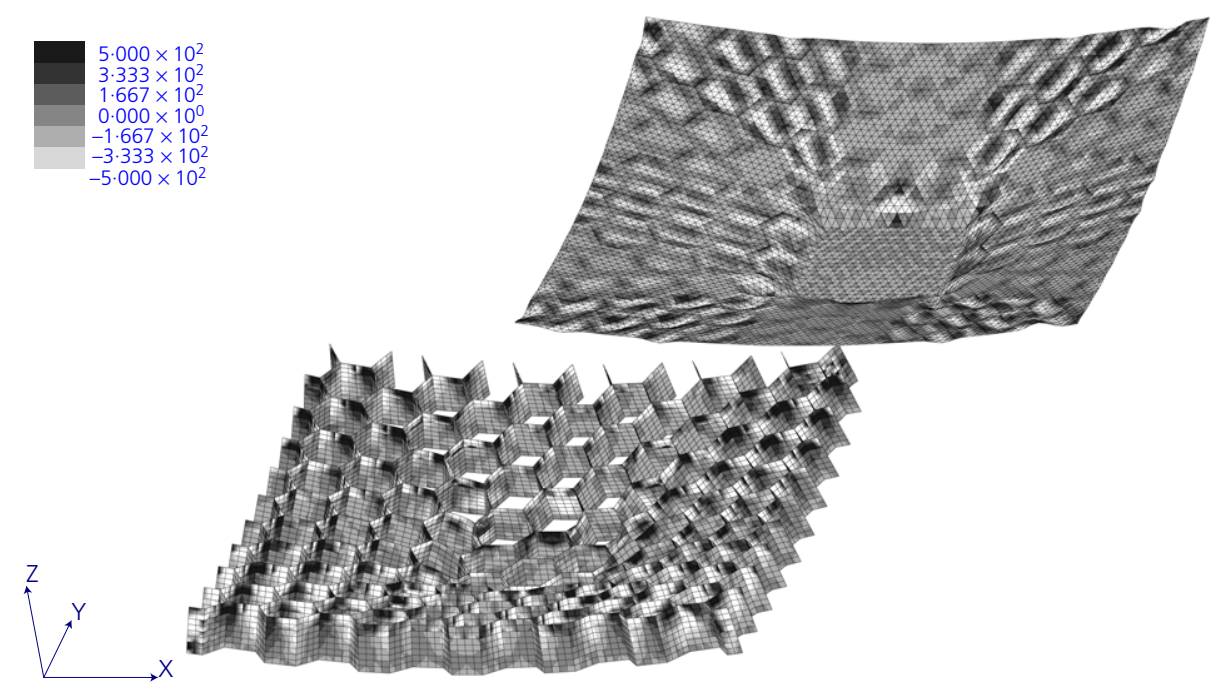

(b)
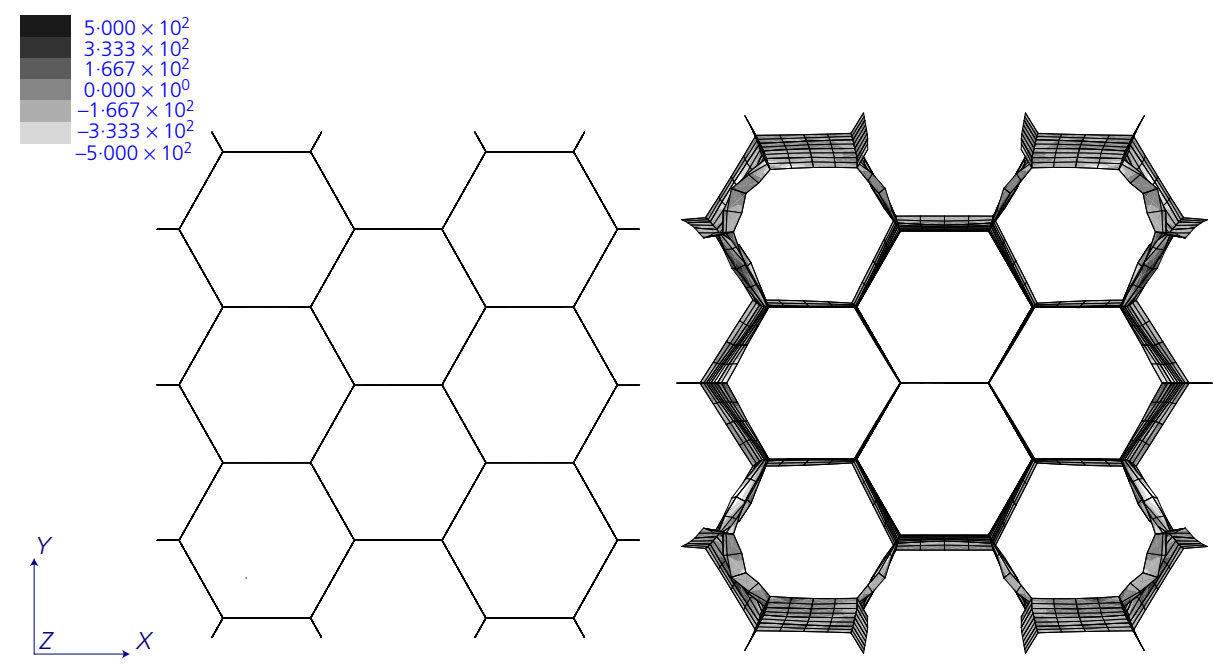

(c)

Figure 10. Deformed configuration at maximum displacement (a) and contours of local $y$-axis bending moment (b, c) for hexagonal honeycomb configurations subject to PL 
Table 1. Computing wall-clock time and achieved speed-up for partitioned modelling

\begin{tabular}{|c|c|c|c|c|}
\hline Model configuration & Wall-clock time: $\min$ & Normalised wall-clock time: \% & Percentage reduction & SF: dimensionless \\
\hline \multicolumn{5}{|c|}{ Base model-1 (BM-1) - 43200 elements, 156842 nodes, 784210 DOF } \\
\hline $\mathrm{MM}$ & 4472 & $100 \cdot 00$ & 0.00 & 1.00 \\
\hline PM-4 & 2166 & $48 \cdot 45$ & $51 \cdot 55$ & $2 \cdot 06$ \\
\hline PM-9 & 1556 & $34 \cdot 79$ & $65 \cdot 21$ & $2 \cdot 87$ \\
\hline PM-16 & 1233 & $27 \cdot 58$ & $72 \cdot 42$ & $3 \cdot 63$ \\
\hline PM-25 & 1177 & $26 \cdot 31$ & 73.69 & 3.80 \\
\hline PM-36 & 1225 & $27 \cdot 40$ & $71 \cdot 68$ & $3 \cdot 65$ \\
\hline PM-49 & 1351 & $30 \cdot 21$ & $69 \cdot 68$ & $3 \cdot 31$ \\
\hline PM-64 & 1445 & $32 \cdot 32$ & $67 \cdot 68$ & 3.09 \\
\hline \multicolumn{5}{|c|}{ Base model-2 (BM-2) - 230400 elements, 882122 nodes, 4410610 DOF } \\
\hline $\mathrm{MM}$ & 36454 & $100 \cdot 00$ & 0.00 & 1.00 \\
\hline PM-4 & 20252 & $55 \cdot 56$ & $44 \cdot 44$ & $1 \cdot 80$ \\
\hline PM-9 & 13113 & $35 \cdot 97$ & 64.03 & $2 \cdot 78$ \\
\hline PM-16 & 10659 & $29 \cdot 24$ & $70 \cdot 76$ & 3.42 \\
\hline PM-25 & 9182 & $25 \cdot 19$ & $74 \cdot 81$ & 3.97 \\
\hline PM-36 & 8210 & $22 \cdot 52$ & $77 \cdot 48$ & 4.44 \\
\hline PM-49 & 7579 & $20 \cdot 79$ & $79 \cdot 21$ & 4.81 \\
\hline PM-64 & 10269 & $28 \cdot 17$ & 71.83 & 3.55 \\
\hline
\end{tabular}

Bold values show specific configurations that achieve optimal performance

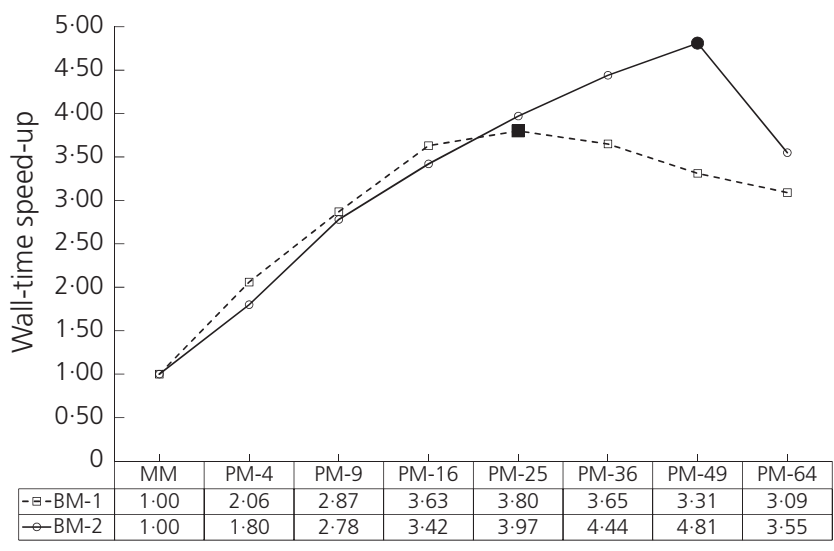

Figure 11. Wall-clock time speed-up for different domain-partitioning configurations of large-scale sandwich panels

where $t_{\mathrm{mono}}$ and $t_{p}$ are the wall-clock times associated with the monolithic and the partitioned models. The absolute and relative wall-clock times as well as the speed-up factors for the adopted set of partitioned models are provided in Table 1 and the speed-up factor is also graphically presented in Figure 11. These results indicate an initially increasing speed-up for an increasing number of child partitions, with a maximum value of 4.81 achieved by BM-2 at 49 partitions, compared with 3.80 achieved by BM-1 at 25 partitions. Clearly, the maximum achieved speed-up is substantially larger and corresponds to a different partitioning configuration when a high mesh density is employed, as with BM-2, which emphasises the particular computational benefits of the partitioned approach for highfidelity modelling.
The progressively decreasing speed-up rate observed for an increasing number of child partitions, ultimately leading to a descending branch, is indicative of an efficiency degradation of the domain-decomposition approach in the case of excessive partitioning. This is due to an increasing communication overhead between parallel processors, which significantly impacts the parallelisation efficiency, ultimately leading to slowdown (Jokhio and Izzuddin, 2015). Moreover, physical limitations imposed by the number of CPUs available in a single computing node of the HPC system require the distribution of models with a very large number of partitions to multiple nodes, as in the case of PM-64, which further encumbers the interprocessor communication. A qualitative guide for establishing the optimal partitioning configuration is proposed here based on the nodal ratio $\mathrm{NR}_{p}$, which is defined for a configuration with $p$ child partitions as

14. $\mathrm{NR}_{p}=\frac{N_{\mathrm{b}}^{(p)}}{n_{i}^{(p)}}=\frac{N_{\mathrm{b}}^{(p)}}{n^{(p)}-n_{\mathrm{b}}^{(p)}}$

where $N_{\mathrm{b}}^{(p)}$ is the number of nodes along the partition boundary at the parent level, $n^{(p)}$ the average total number of nodes of a child partition, $n_{\mathrm{b}}^{(p)}$ the average number of nodes on the child partition boundary and $n_{i}^{(p)}$ the average number of internal nodes in the child partition domain.

Under idealised process parallelisation conditions, where CPUs have identical specifications, communication overhead and processing time for problems of the same size, it is found that optimal efficiency is achieved at $\mathrm{NR}_{p}=1$ for partitioned modelling of all-metal sandwich panels, which corresponds to the distribution of equivalently sized problems over all CPUs. 
Table 2. Nodal quantities for partitioned modelling configurations

\begin{tabular}{|c|c|c|c|c|c|}
\hline Model configuration & $n$ & $n_{\mathrm{b}}$ & $n_{i}=n-n_{\mathrm{b}}$ & $N_{\mathrm{b}}$ & Nodal ratio $\mathrm{NR}=n_{i} / N_{\mathrm{b}}$ \\
\hline \multicolumn{6}{|c|}{ Base model-1 (BM-1) - 43200 elements, 156842 nodes, 784210 DOF } \\
\hline PM-4 & 56282 & 1140 & 55142 & 4136 & $13 \cdot 3$ \\
\hline PM-9 & 25122 & 760 & 24362 & 5512 & $4 \cdot 4$ \\
\hline PM-16 & 16230 & 608 & 15622 & 6888 & $2 \cdot 3$ \\
\hline PM-25 & 9122 & 456 & 8666 & 8264 & 1.0 \\
\hline PM-36 & 6092 & 460 & 5632 & 9590 & $0 \cdot 6$ \\
\hline PM-49 & 6092 & 460 & 5632 & 10944 & $0 \cdot 5$ \\
\hline PM-64 & 4098 & 304 & 3794 & 10232 & $0 \cdot 4$ \\
\hline \multicolumn{6}{|c|}{ Base model-2 (BM-2) - 230400 elements, 882122 nodes, 4410610 DOF } \\
\hline PM-4 & 221237 & 2820 & 218417 & 8454 & $25 \cdot 8$ \\
\hline PM-9 & 98642 & 1880 & 96762 & 11264 & $8 \cdot 6$ \\
\hline PM-16 & 63282 & 1504 & 61778 & 14070 & $4 \cdot 4$ \\
\hline PM-25 & 35738 & 1128 & 34610 & 16872 & $2 \cdot 1$ \\
\hline PM-36 & 24897 & 940 & 23957 & 19720 & $1 \cdot 2$ \\
\hline PM-49 & 24897 & 940 & 23957 & 22528 & $1 \cdot 1$ \\
\hline PM-64 & 16010 & 752 & 15258 & 25254 & 0.6 \\
\hline
\end{tabular}

Bold values show specific configurations that achieve optimal performance

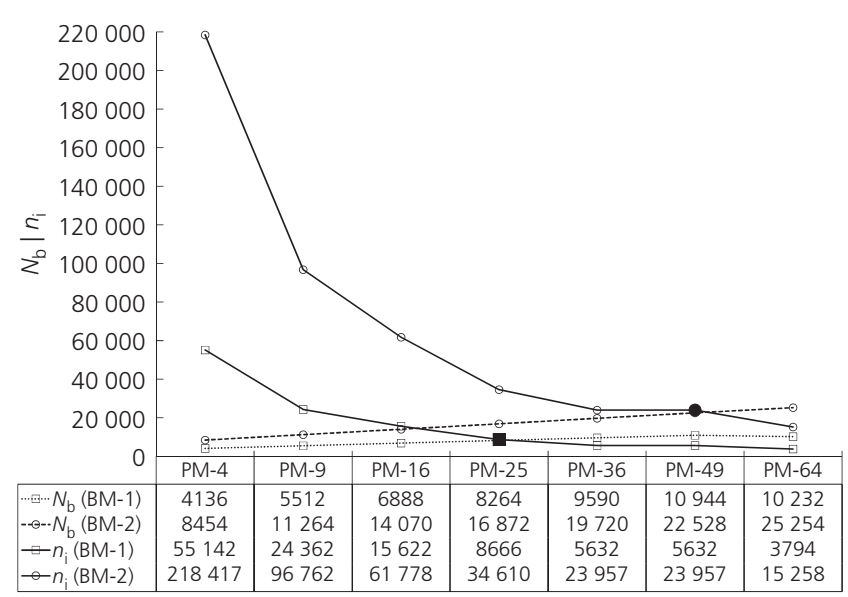

Figure 12. Parent and child partition process nodal balance for optimal efficiency of large-scale sandwich panels domain partitioning

As presented in Table 2 and shown in Figure 12, optimal speed-up is achieved for a different number of child partitions for the two levels of discretisation, BM-1 and BM-2, which leads to a maximum speed-up at 25 and 49 child partitions, respectively. For values of the nodal ratio less than 1, the computing demand is dictated by the child partitions, while for values greater than 1 , by the number of nodes on the partition boundary at the parent level, thereby compromising the essence of the domain-decomposition method. This outcome can even be better illustrated by considering the extreme case where the number of partitions becomes the same as the number of FEs employed for the domain discretisation, where

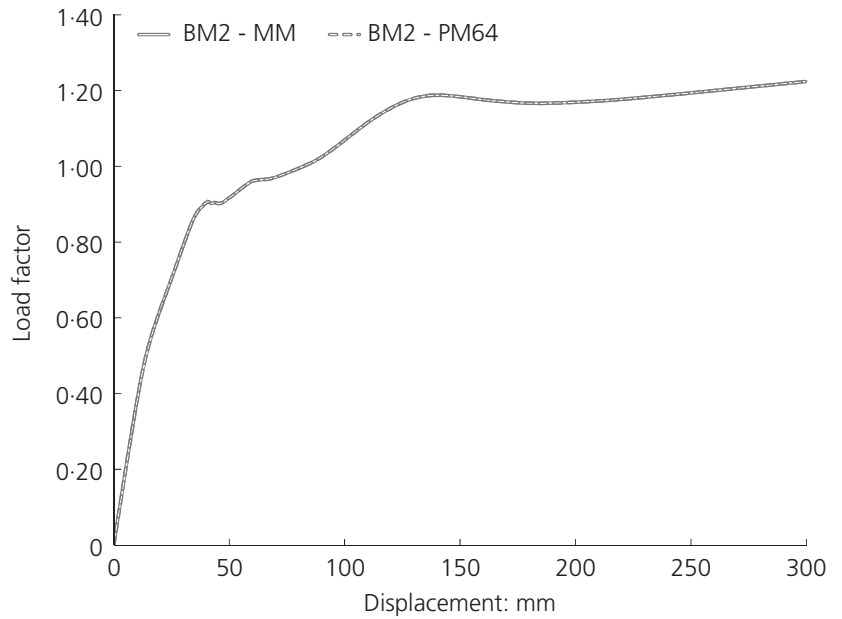

Figure 13. Non-linear response comparison for monolithic and partitioned high-fidelity models of large-scale rectangularhoneycomb-core sandwich panels

the partitioned model effectively degenerates into the monolithic model, but with a significant communication overhead, thus achieving virtually zero speed-up.

The comparison of the non-linear response of BM-2 obtained for a monolithic model and a partitioned configuration with 64 child partitions is illustrated in Figure 13. Evidently, the two configurations exhibit a virtually identical non-linear equilibrium path, which highlights the capability of domain partitioning to achieve substantial computational benefits, without compromising the accuracy and convergence rate of the monolithic approach, as discussed previously in Section 3. 


\section{Conclusions}

This paper presents a high-fidelity methodology for the nonlinear analysis of large-scale metal sandwich panels, coupled with a partitioned modelling approach which allows for scalable parallel processing of individual partitions in HPC systems with distributed memory.

The high-fidelity methodology employs non-linear co-rotational shell FEs, attaining optimal performance through a hierarchic optimisation approach that enables membrane, bending and shear locking phenomena to be addressed. This allows for the accurate capturing of local buckling and material nonlinearities for large-scale sandwich systems in the large displacement range, which is crucial due to the increased response sensitivity of such systems to the employed level of discretisation detail and to the magnitude of geometric imperfections.

The partitioned modelling approach leads to significant computational benefits when the high-fidelity modelling strategy is employed for large-scale sandwich systems, leading to virtually $80 \%$ reduction of the associated wall-clock time, while enabling for memory bottlenecks to be effectively overcome. The process parallelisation efficiency is enhanced with increased partitioning, with the maximum speed-up achieved for a balanced DOF distribution in the parent and individual child partition processes. Excessive partitioning leads to a deteriorating performance, due to excesses both in the non-linear solution procedure and the communication overhead between parallel processors, with a consequent increase in computing time.

The potential and significance of the proposed method are highly profound in cases where computing resources limitations render non-linear analysis prohibitively demanding or impossible. This applies in particular to the accurate non-linear assessment of all-metal sandwich panels subject to extreme static or dynamic loading, where the computational demands of the conventional high-fidelity modelling approach can be prohibitive, particularly for large-scale systems with interacting panels.

\section{Acknowledgement}

The support of the HPC Services at Imperial College London is gratefully acknowledged by the authors.

\section{REFERENCES}

Allen HG (1969) Analysis and Design of Structural Sandwich Panels. Pergamon, Oxford, UK.

CORe, SAND (2013) Best Practice Guide for Sandwich Structures in Marine Applications. Coordination Action on Advanced Sandwich Structures in the Transport Industries, Under European Commission Contract No. FP6-506330, S.A.N.D. CORe, Newcastle upon Tyne, UK.

Cote F, Deshpande V, Fleck N and Evans A (2004) The out-of-plane compressive behavior of metallic honeycombs. Materials Science and Engineering: A 380(1-2): 272-280.
Gibson LJ and Ashby MF (1999) Cellular Solids: Structure and Properties. Cambridge University Press, Cambridge, UK. Izzuddin BA (1991) Nonlinear Dynamic Analysis of Framed Structures. $\mathrm{PhD}$ thesis, Imperial College, University of London, London, UK. Izzuddin B and Liang Y (2016) Bisector and zero-macrospin co-rotational systems for shell elements. International Journal for Numerical Methods in Engineering 105(4): 286-320.

Izzuddin BA and Liang Y (2017) A hierarchic optimisation approach towards locking-free shell finite elements. Computers \& Structures, https://doi.org/10.1016/j.compstruc.2017.08.010.

Jokhio GA and Izzuddin BA (2015) A dual super-element domain decomposition approach for parallel nonlinear finite element analysis. International Journal for Computational Methods in Engineering Science and Mechanics 16(3): 188-212.

Kee Paik J, Thayamballi AK and Sung Kim G (1999) The strength characteristics of aluminum honeycomb sandwich panels. ThinWalled Structures 35(3): 205-231.

Kolsters $\mathrm{H}$ and Zenkert D (2006a) Buckling of laser-welded sandwich panels. Part 1: elastic buckling parallel to the webs. Proceedings of the Institution of Mechanical Engineers, Part M: Journal of Engineering for the Maritime Environment 220(2): 67-79.

Kolsters $\mathrm{H}$ and Zenkert D (2006b) Buckling of laser-welded sandwich panels. Part 2: elastic buckling normal to the webs. Proceedings of the Institution of Mechanical Engineers, Part M: Journal of Engineering for the Maritime Environment 220(2): 81-94.

Kolsters H and Zenkert D (2010) Buckling of laser-welded sandwich panels: ultimate strength and experiments. Proceedings of the Institution of Mechanical Engineers, Part M: Journal of Engineering for the Maritime Environment 224(1): 29-45.

Liang $S$ and Chen $H$ (2006) Investigation on the square cell honeycomb structures under axial loading. Composite Structures 72(4): 446-454.

López Jiménez F and Triantafyllidis N (2013) Buckling of rectangular and hexagonal honeycomb under combined axial compression and transverse shear. International Journal of Solids and Structures 50(24): 3934-3946.

Plantema FJ (1966) Sandwich Construction. Wiley, New York, NY, USA. Zenkert D (1995) An Introduction to Sandwich Construction. EMAS Ltd, London, UK.

Zhang J and Ashby M (1992) The out-of-plane properties of honeycombs. International Journal of Mechanical Sciences 34(6): 475-489.

Zok FW, Rathbun H, He M et al. (2005) Structural performance of metallic sandwich panels with square honeycomb cores. Philosophical Magazine 85(26-27): 3207-3234.

\section{How can you contribute?}

To discuss this paper, please email up to 500 words to the editor at journals@ice.org.uk. Your contribution will be forwarded to the author(s) for a reply and, if considered appropriate by the editorial board, it will be published as discussion in a future issue of the journal.

Proceedings journals rely entirely on contributions from the civil engineering profession (and allied disciplines). Information about how to submit your paper online is available at www.icevirtuallibrary.com/page/authors, where you will also find detailed author guidelines. 